Anomaly freedom in Seiberg-Witten noncommutative gauge theories

This article has been downloaded from IOPscience. Please scroll down to see the full text article.

JHEP07(2003)068

(http://iopscience.iop.org/1126-6708/2003/07/068)

View the table of contents for this issue, or go to the journal homepage for more

Download details:

IP Address: 147.96.14.16

The article was downloaded on 08/08/2013 at $12: 48$

Please note that terms and conditions apply. 


\title{
Anomaly freedom in Seiberg-Witten noncommutative gauge theories
}

\author{
Friedemann Brandt \\ Max-Planck-Institute for Mathematics in the Sciences \\ Inselstraße 22-26, D-04103 Leipzig, Germany \\ E-mail: fbrandt@mis.mpg.de

\section{Carmelo P. Martín} \\ Departamento de Física Teórica I, Facultad de Ciencias Físicas \\ Universidad Complutense de Madrid, 28040 Madrid, Spain \\ E-mail: carmelo@elbereth.fis.ucm.es \\ Fernando Ruiz Ruiz \\ Departamento de Física Teórica I, Facultad de Ciencias Físicas \\ Universidad Complutense de Madrid, 28040 Madrid, Spain \\ E-mail: t630aeneas.fis.ucm.es
}

ABSTRACT: We show that noncommutative gauge theories with arbitrary compact gauge group defined by means of the Seiberg-Witten map have the same one-loop anomalies as their commutative counterparts. This is done in two steps. By explicitly calculating the $\epsilon^{\mu_{1} \mu_{2} \mu_{3} \mu_{4}}$ part of the renormalized effective action, we first find the would-be one-loop anomaly of the theory to all orders in the noncommutativity parameter $\theta^{\mu \nu}$. And secondly we isolate in the would-be anomaly radiative corrections which are not BRS trivial. This gives as the only true anomaly occurring in the theory the standard Bardeen anomaly of commutative spacetime, which is set to zero by the usual anomaly cancellation condition.

KeYwords: BRST Symmetry, Anomalies in Field and String Theories. 


\section{Contents}

1. Introduction 1

2. The model, notation and conventions 3

3. Form of the noncommutative anomaly 6

4. BRS triviality of $\theta$-dependent contributions 10

5. Conclusion 11

A. Useful integrals 12

B. Proof of eq. 4.1 13

B.1 Computation of $\mathcal{B}_{3}$ and $\mathcal{B}_{4}$

B.2 Calculation of $\mathcal{B}_{5}$ and $\mathcal{B}_{6}$

\section{Introduction}

It is a well known fact that not all relevant gauge groups in particle physics are consistent with the Moyal product of noncommutative field theory. An example of this is provided by the Moyal product $A_{\mu}(x) \star A_{\nu}(x)$ of two $\mathrm{SU}(N)$ Lie algebra valued gauge fields $A_{\mu}(x)$ and $A_{\nu}(x)$. It is clear that such product does not lie in the $\mathrm{SU}(N)$ Lie algebra but in a representation of its enveloping algebra, so $A_{\mu}(x)$ can not be regarded as a truly noncommutative $\mathrm{SU}(N)$ gauge field. This makes it difficult to formulate, even classically, noncommutative extensions of some physically relevant gauge theories like e.g. the standard model. A way to circumvent this problem [1, 2] is to build noncommutative gauge and matter fields from ordinary ones by means of the Seiberg-Witten map [3]. Using this approach, classical noncommutative gauge theories have been constructed for arbitrary compact groups [1]-[0] and noncommutative gauge theories with $\mathrm{SU}(5)$ and $\mathrm{SO}(10)$ gauge groups have been constructed in ref. [13]. Furthermore, a noncommutative standard model has been formulated in ref. [6] and some of its phenomenological consequences have been explored in a number of papers [7]-[12]. Many of these noncommutative gauge theories, among them the noncommutative standard model, involve chiral fermions, so the corresponding classical gauge symmetry may be broken by quantum corrections. In other words, an anomaly may occur and the resulting quantum theory may then become inconsistent. To study the consistency of quantum noncommutative gauge theories defined by means of the Seiberg-Witten map, 
it is therefore necessary to study whether new types of anomalies occur - i.e. anomalies which do not appear in ordinary commutative spacetime and hence that may require additional anomaly cancellation conditions.

In refs. [14, 15, 16] it has been shown that for Yang-Mills type gauge theories with arbitrary semisimple gauge groups the only nontrivial solution to the anomaly consistency condition is the usual Bardeen anomaly, regardless of whether or not the theory is Lorentz invariant or renormalizable by power counting. This result readily applies to gauge noncommutative field theories constructed by means of the Witten-Seiberg map, since, as far as these matters are concerned, the presence of a noncommutative matrix parameter $\theta^{\mu \nu}$ with mass dimension -2 only precludes Lorentz invariance and power-counting renormalizability. Thus, for noncommutative gauge theories with semisimple gauge groups, there are no $\theta^{\mu \nu}$-dependent anomalies and any $\theta^{\mu \nu}$-dependent breaking of the BRS identity, being cohomologically trivial, can be set to zero by adding appropriate counterterms to the effective action. Note that the addition of these $\theta^{\mu \nu}$-dependent counterterms to the effective action makes sense within the framework of effective field theory, but this agrees with the observation that noncommutative field theories defined by means of the Seiberg-Witten map should be considered as effective field theories [13, 17]. All the above implies that no anomalous $\theta^{\mu \nu}$-dependent terms should occur in the Green functions of noncommutative theories with semisimple gauge groups, a fact that has been proved to hold true at order one in $\theta^{\mu \nu}$ for the three-point function of the gauge field and a simple gauge group by explicit computation of the appropriate Feynman diagrams [18].

The situation is very different if the gauge group is not semisimple. In this case, the consistency condition for gauge anomalies has other nontrivial solutions besides Bardeen's anomaly. In particular, in four dimensions and if the gauge group is $G \times U(1)_{\mathrm{Y}}$, with $G$ a semisimple gauge group, the additional nontrivial solutions are of the form

$$
\int d^{4} x c \mathrm{I}_{\mathrm{inv}}\left[f_{\mu \nu}, G_{\mu \nu}\right]
$$

Here matter fields have been integrated out, $c$ is the $\mathrm{U}(1)_{\mathrm{Y}}$ ghost field and $\mathrm{I}_{\mathrm{inv}}\left[f_{\mu \nu}, G_{\mu \nu}\right]$ is a gauge invariant function of the $\mathrm{U}(1)_{Y}$ field strength $f_{\mu \nu}$, the $G$ field strength $G_{\mu \nu}$ and their covariant derivatives. Note that there are infinitely many candidate anomalies of this type since neither power counting nor Lorentz invariance are available to reduce the number of invariants $\mathrm{I}_{\mathrm{inv}}\left[f_{\mu \nu}, G_{\mu \nu}\right]$. Furthermore, when the gauge group contains more than one abelian factor, there are additional candidate anomalies of yet another type [14, 15, 16. The purpose of this paper is to investigate whether anomalies of these types occur in noncommutative gauge theories with nonsemisimple gauge groups defined through the Seiberg-Witten map. This is not a trivial question and has far reaching implications. Indeed, did solutions of type (1.1) occur in perturbation theory, the corresponding quantum gauge theory would be anomalous, the anomaly being $\theta^{\mu \nu}$-dependent. To remove the resulting anomaly and render the quantum theory consistent, one would then have to impose constraints on the fermions hypercharges. A conspicuous instance of a model with such a gauge group for which this point should be cleared is the noncommutative standard model [6]. 
In this paper we will prove that, for a noncommutative field theory with arbitrary compact gauge group defined by means of the Seiberg-Witten map, the only anomaly that occurs at one loop (hence, to all orders in perturbation theory, if one assumes the existence of a nonrenormalization theorem for the anomaly) is the usual Bardeen anomaly. The paper is organized as follows. In section 2 we fix the notation, define the chiral BRS transformations and use the Seiberg-Witten map to classically define the noncommutative model. Section 3 uses dimensional regularization to explicitly compute the $\epsilon^{\mu_{1} \mu_{2} \mu_{3} \mu_{4}}$ part of the renormalized effective action. This yields a complicated power series in the noncommutativity parameter $\theta^{\mu \nu}$, of which the term of order zero is the usual Bardeen anomaly of commutative field theory. In section 1 we show that all terms in this series of order one or higher in $\theta^{\mu \nu}$ are cohomologically trivial with respect to the chiral BRS operator and find the counterterm that removes them from the renormalized effective action. Section 5 contains our conclusions. We postpone to two appendices some very technical points of our arguments. Let us emphasize that in this paper we will only discuss gauge anomalies - see refs. [19, 20] for related work on the rigid axial anomaly.

\section{The model, notation and conventions}

Let us consider a compact nonsemisimple gauge group $G=G_{1} \times \cdots \times G_{N}$, with $G_{i}$ a simple compact group if $i=1, \ldots, s$ and an abelian group if $i=s+1, \ldots, N$. We may assume without loss of generality that the abelian factors come with irreducible representations, which of course are one-dimensional. Let us denote by $\psi_{i_{1} \cdots i_{s}}$ a Dirac field on ordinary Minkowski spacetime carrying an arbitrary unitary irreducible representation of the Lie algebra of $G$. Since the abelian factors come with one-dimensional representations, the indices in the Dirac field $\psi_{i_{1} \cdots i_{s}}$ correspond to the simple factors. From now on we will collectively denote the "simple" indices $\left(i_{1} \cdots i_{s}\right)$ by the multi-index $I$. The corresponding vector potential $v_{\mu}$ on Minkowski spacetime in the representation of the Lie algebra carried by $\psi_{I}$ will have the following decomposition in terms of the gauge fields $a_{\mu}^{k}$ and $a_{\mu}^{l}$ associated to the factors of the group $G$

$$
v_{\mu}=\sum_{k=1}^{s} g_{k}\left(a_{\mu}^{k}\right)^{a}\left(T^{k}\right)^{a}+\sum_{l=s+1}^{N} g_{l} a_{\mu}^{l} T^{l} .
$$

Here $g_{k}$ and $g_{l}$ are the coupling constants and $\left\{\left(T^{k}\right)^{a}, T^{l}\right\}$, with $a=1, \ldots$, $\operatorname{dim} G_{k}$ for every $k=1, \ldots, s$ and $l=s+1, \ldots, N$, stand for the generators of the $G$ Lie algebra in the unitary irreducible representation under consideration. As usual, a sum over $a$ is understood. The matrix elements $I J$ of these generators are always of the form

$$
\begin{aligned}
\left(T^{k}\right)_{I J}^{a} & =\delta_{i_{1} j_{1}} \cdots\left(T^{k}\right)_{i_{k} j_{k}}^{a} \cdots \delta_{i_{s} j_{s}} \\
T_{I J}^{l} & =\delta_{i_{1} j_{1}} \cdots \delta_{i_{s} j_{s}} Y^{l},
\end{aligned}
$$

$\left(T^{k}\right)_{i_{k} j_{k}}^{a}$ are the matrix elements of the generator $\left(T^{k}\right)^{a}$ of the Lie algebra of the factor $G_{k}$ in some given irreducible representation. Given any two generators $\left(T^{k}\right)_{I J}^{a}$ and $\left(T^{k^{\prime}}\right)_{I J}^{a^{\prime}}$ as 
above we define the trace operation $\operatorname{Tr}$ as

$$
\begin{aligned}
\operatorname{Tr}\left(T^{k}\right)^{a}\left(T^{k^{\prime}}\right)^{a^{\prime}} & =\left(T^{k}\right)_{I J}^{a}\left(T^{k^{\prime}}\right)_{J I}^{a_{J}^{\prime}} \\
& =\delta_{i_{1} j_{1}} \cdots\left(T^{k}\right)_{i_{k} j_{k}}^{a} \cdots \delta_{i_{s} j_{s}} \delta_{j_{1} i_{1}} \cdots\left(T^{k^{\prime}}\right)_{j_{k^{\prime}} i_{k^{\prime}}}^{a^{\prime}} \cdots \delta_{j_{s} i_{s}} .
\end{aligned}
$$

The ghost field $\lambda$ associated to $v_{\mu}$, also in the representation furnished by $\psi_{I}$, is

$$
\lambda=\sum_{k=1}^{s} g_{k}\left(\lambda^{k}\right)^{a}\left(T^{k}\right)^{a}+\sum_{l=s+1}^{N} g_{l} \lambda^{l} T^{l},
$$

with $\left(\lambda^{k}\right)^{a}$ and $\lambda^{l}$ being the ghost fields for the factors in $G$. Now we consider the theory that arises from chirally coupling, say left-handedly, the fermion field $\psi_{I}$ to the gauge field $v_{\mu}$. The fermionic part of the corresponding classical action reads

$$
S^{\text {fermion }}=\int d^{4} x \bar{\psi}_{I} i \hat{D}(v)_{I J} \psi_{J}
$$

with $\bar{\psi}_{I}=\psi_{I}^{\dagger} \gamma^{0}$ and

$$
\hat{D}(v)_{I J} \psi_{J}=\delta_{I J} \not \partial \psi_{J}+\psi_{I J} \mathrm{P}_{-} \psi_{J} .
$$

Here $\mathrm{P}_{-}$is the left-handed chiral projector, given by

$$
\mathrm{P}_{ \pm}=\frac{1}{2}\left(1 \pm \gamma_{5}\right) \quad \gamma_{5}=-i \gamma^{0} \gamma^{1} \gamma^{2} \gamma^{3}
$$

the gamma matrices $\gamma^{\mu}$ being defined by $\left\{\gamma^{\mu}, \gamma^{\nu}\right\}=2 \eta^{\mu \nu}$ and the convention for the Minkowski metric $\eta_{\mu \nu}$ being $\eta_{\mu \nu}=\operatorname{diag}(+,-,-,-)$. This action is invariant under the chiral BRS transformations

$$
s v_{\mu}=\partial_{\mu} \lambda+\left[v_{\mu}, \lambda\right] \quad s \psi=-\lambda \mathrm{P}_{-} \psi \quad s \bar{\psi}=\bar{\psi} \lambda \mathrm{P}_{+} \quad s \lambda=-\lambda \lambda .
$$

As usual, the BRS operator $s$ commutes with $\partial_{\mu}$, satisfies the anti-Leibniz rule and is nilpotent, i.e. $s^{2}=0$.

To construct the noncommutative extension of the ordinary gauge theory defined by

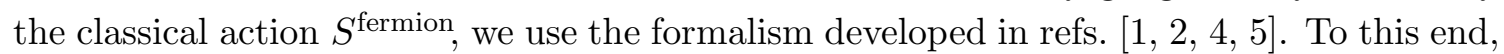
we first define the noncommutative gauge field $V_{\mu}$, the noncommutative spinor field $\Psi_{I}$ and the noncommutative ghost field $\Lambda$ in terms of their ordinary counterparts $v_{\mu}, \psi_{I}$ and $\lambda$ by using the Seiberg-Witten map [3]. This is done as follows. The fields $V_{\mu}=V_{\mu}[v ; \theta], \Psi_{I}=$ $\Psi_{I}[\psi, v ; \theta]$ and $\Lambda=\Lambda[\lambda, v ; \theta]$ are formal power series in $\theta^{\mu \nu}$, with coefficients depending on the ordinary fields and their derivatives, that take values in the representation of the enveloping algebra of the Lie algebra of the group $G$ furnished by the ordinary Dirac field $\psi_{I}$ and solve the Seiberg-Witten equations

$$
s_{\star} V_{\mu}=s V_{\mu} \quad s_{\star} \Psi=s \Psi \quad s_{\star} \Lambda=s \Lambda
$$

subject to the boundary conditions

$$
V_{\mu}[v ; \theta=0]=v_{\mu} \quad \Psi_{I}[v, \psi ; \theta=0]=\psi_{I} \quad \Lambda[\lambda, v ; \theta=0]=\lambda .
$$


In eq. (2.3) $s$ is the ordinary BRS operator of eq. (2.2), while $s_{\star}$ denotes the noncommutative BRS chiral operator, whose action on the noncommutative fields is given by

$$
s_{\star} V_{\mu}=\partial_{\mu} \Lambda+\left[V_{\mu}, \Lambda\right]_{\star} \quad s_{\star} \Psi=-\Lambda \star \mathrm{P}_{-} \Psi \quad s_{\star} \Lambda=-\Lambda \star \Lambda .
$$

The commutator $[f, g]_{\star}$ stands for

$$
[f, g]_{\star}=f \star g-g \star f,
$$

with $f \star g$ the Moyal product of functions on Minkowski spacetime, defined for arbitrary $f$ and $g$ by

$$
(f \star g)(x)=\int \frac{d^{4} p}{(2 \pi)^{4}} \int \frac{d^{4} q}{(2 \pi)^{4}} e^{-i(p+q) x} e^{-\frac{i}{2} \theta^{\alpha \beta} p_{\alpha} q_{\beta}} \tilde{f}(p) \tilde{q}(q),
$$

$\tilde{f}(p)$ and $\tilde{g}(q)$ being the Fourier transforms of $f$ and $g$. For the noncommutative field $\Psi_{I}$ we further demand it to be linear in $\psi_{I}$, so that

$$
\Psi_{\alpha I}=\left(\delta_{I J} \delta_{\alpha \beta}+M\left[v, \partial, \gamma, \gamma_{5} ; \theta\right]_{\alpha \beta I J}\right) \psi_{\beta J},
$$

where $\alpha$ and $\beta$ are Dirac indices. Note that, in accordance with the boundary condition for $\Psi_{I}[\psi, v ; \theta]$, the differential operator $M\left[v, \partial, \gamma, \gamma_{5} ; \theta\right]_{\alpha \beta I J}$ vanishes at $\theta=0$. Taking $\Psi_{i}$ linear in $\psi_{I}$, as in eq. (2.6), is always possible [21] and is the natural choice within the framework of noncommutative geometry [17]. Once the noncommutative fields have been defined, one considers the following noncommutative classical action

$$
S_{\mathrm{nc}}^{\text {fermion }}=\int d^{4} x \bar{\Psi}_{I} \star i \hat{D}(V)_{I J} \Psi_{J}
$$

where $\bar{\Psi}_{I}=\bar{\Psi}_{I}^{\dagger} \gamma^{0}$ and

$$
\hat{D}(V)_{I J} \Psi_{J}=\delta_{I J} \not \partial \Psi_{J}+V_{I J} \star \mathrm{P}_{-} \Psi_{J} .
$$

We stress that the noncommutative fields are functions of ordinary fields as given by the Seiberg-Witten map and hence the noncommutative action is also a functional of these. Furthermore, the noncommutative action $S_{\mathrm{nc}}^{\text {fermion }}$ is invariant under the ordinary chiral BRS transformations in eq. (2.2) since, by definition of the Seiberg-Witten map,

$$
s S_{\mathrm{nc}}^{\text {fermion }}=s_{\star} S_{\mathrm{nc}}^{\text {fermion }}
$$

and, by construction,

$$
s_{\star} S_{\text {nc }}^{\text {fermion }}=0 .
$$

The effective action $\Gamma[v ; \theta]$ of the noncommutative theory is formally defined by

$$
\begin{aligned}
& \Gamma[v ; \theta]=-i \ln Z[v ; \theta] \\
& Z[v ; \theta]=\mathcal{N} \int[d \bar{\psi}][d \psi] \exp \left(i S_{\mathrm{nc}}^{\mathrm{fermion}}\right)
\end{aligned}
$$


with $\mathcal{N}$ a normalization constant chosen so that $Z[v=0 ; \theta]=1$, i.e.

$$
\mathcal{N}^{-1}=\int[d \bar{\psi}][d \psi] \exp \left(\int d^{4} x \bar{\psi} i \not \partial \psi\right)
$$

and $[d \bar{\psi}][d \psi]$ the measure for ordinary fermion fields. Also formally, the invariance of $S_{\mathrm{nc}}^{\text {fermion }}$ under $s$ leads to the invariance of $\Gamma[v ; \theta]$ under ordinary gauge transformations of $v_{\mu}$. The problem is that all this is formal since defining the effective action requires renormalization. The question that should really be addressed is whether it is possible to define a renormalized effective action $\Gamma^{\mathrm{ren}}[v ; \theta]$ invariant under $s$. Were this the case, the theory would be anomaly free. In this paper we provide an answer in the negative and show that the anomaly has the same form as for the ordinary, i.e. commutative theory.

\section{Form of the noncommutative anomaly}

In this section we use dimensional regularization to define a renormalized effective action $\Gamma^{\mathrm{ren}}[v ; \theta]$ and find a closed expression for the anomaly $s \Gamma^{\mathrm{ren}}[v ; \theta]$ in terms of the noncommutative fields $V_{\mu}$ and $\Lambda$. To dimensionally regularize the theory, we consider the action

$$
S_{\mathrm{nc}}^{\mathrm{reg}}=\int d^{2 \omega} x \bar{\Psi}_{I} \star i\left(\delta_{I J} \not \partial \Psi_{J}+\bar{\gamma}^{\mu} V_{\mu I J} \star \mathrm{P}_{-} \Psi_{J}\right),
$$

first introduced in the context of noncommutative gauge theories in ref. [18] for $\mathrm{U}(N)$ theories and theories with simple groups. Here we use dimensional regularization á la Breitenlohner and Maison [22]. We will use the notation in that reference, in which 4dimensional objects are denoted with bars $\left(\bar{g}^{\mu}{ }_{\mu}=4\right)$ and evanescent or $(2 \omega-4)$-dimensional quantities are denoted with hats $\left(\hat{g}_{\mu}^{\mu}=2 \omega-4\right)$. The dimensionally regularized partition function $Z^{\text {reg }}[v ; \theta]$ is defined as the sum of the dimensionally regularized Feynman diagrams generated by the path integral

$$
Z^{\mathrm{reg}}[v ; \theta]=\mathcal{N} \int[d \bar{\psi}][d \psi] \exp \left(i S_{\mathrm{nc}}^{\mathrm{reg}}\right) .
$$

In the regularized partition function we perform the change of variables $\psi_{\beta J}, \bar{\psi}_{\beta J} \rightarrow$ $\Psi_{\alpha I}, \bar{\Psi}_{\alpha I}$, with

$$
\begin{aligned}
\Psi_{\alpha I} & =\left(\delta_{I J} \delta_{\alpha \beta}+M\left[v, \partial, \gamma, \gamma_{5} ; \theta\right]_{\alpha \beta I J}\right) \psi_{\beta J} \\
\bar{\Psi}_{\alpha I} & =\left(\delta_{I J} \delta_{\alpha \beta}+\bar{M}\left[v, \partial, \gamma, \gamma_{5} ; \theta\right]_{\alpha \beta I J}\right) \bar{\psi}_{\beta J} \\
{[d \bar{\psi}][d \psi] } & =\operatorname{det}(\mathbb{I}+\bar{M}) \operatorname{det}(\mathbb{I}+M)[d \bar{\Psi}][d \Psi],
\end{aligned}
$$

where the determinants are defined by their diagrammatic expansion in dimensional regularization in powers of $\theta$. Now, in dimensional regularization we have

$$
\operatorname{det}(\mathbb{I}+M)=\operatorname{det}(\mathbb{I}+\bar{M})=1 .
$$

To see this, take e.g. the determinant $\operatorname{det}(\mathbb{I}+M)$ and write it as the partition function

$$
\operatorname{det}(\mathbb{I}+M)=\int[d \bar{\psi}][d \psi] e^{i S[M]}
$$


of a fermion theory with classical action

$$
S[M]=\int d^{2 \omega} x \bar{\psi}(\mathbb{I}+M) \psi .
$$

The propagator of such a theory is the identity and the interaction vertices come from the operator $M\left[v, \partial, \gamma, \gamma_{5} ; \theta\right]$, so the Feynman integrals that enter the diagrammatic expansion of $\operatorname{det}(\mathbb{I}+M)$ are of the form

$$
\int \frac{d^{2 \omega} q}{(2 \pi)^{2 \omega}} q_{\mu_{1}} \cdots q_{\mu_{n}}
$$

Since this integral vanishes in dimensional regularization, eq. (3.4) holds and the change of variables (3.3) gives for the path integral in (3.2)

$$
Z^{\mathrm{reg}}[v ; \theta]=\mathcal{N} \int[d \bar{\Psi}][d \Psi] \exp \left(i S_{\mathrm{nc}}^{\mathrm{reg}}\left[\bar{\Psi}, \Psi, V_{\mu}\right]\right) .
$$

Hence $Z^{\text {reg }}[v ; \theta]$ is a functional of $V_{\mu}$, and so is the regularized effective action

$$
\Gamma^{\mathrm{reg}}[v ; \theta]=-i \ln Z[v ; \theta]^{\mathrm{reg}}=\Gamma^{\mathrm{reg}}[V] .
$$

In other words, the regularized effective action depends on $v_{\mu}$ through $V_{\mu}$.

Eq. (3.6) for $\Gamma^{\mathrm{reg}}[V]$ is to be understood in a diagrammatic sense as the generating functional of 1PI Green functions for the field $V_{\mu}$. That is to say,

$$
i \Gamma^{\mathrm{reg}}[V]=\sum_{n=1}^{\infty} \frac{1}{n !} \int d^{2 \omega} x_{1} \ldots \int d^{2 \omega} x_{n} V_{\mu_{1} I_{1} J_{1}}\left(x_{1}\right) \ldots V_{\mu_{n} I_{n} J_{n}}\left(x_{n}\right) \Gamma_{I_{1} J_{1} \ldots I_{n} J_{n}}^{\mu_{1} \ldots \mu_{n}}\left(x_{1}, \ldots, x_{n}\right),
$$

with

$$
\Gamma_{I_{1} J_{1} \ldots I_{n} J_{n}}^{\mu_{1} \ldots \mu_{n}}\left(x_{1}, \ldots, x_{n}\right)=\left\langle\mathcal{J}_{I_{1} J_{1}}^{\mu_{1}}\left(x_{1}\right) \ldots \mathcal{J}_{I_{n} J_{n}}^{\mu_{n}}\left(x_{n}\right)\right\rangle_{0}^{\mathrm{conn}}
$$

and

$$
\mathcal{J}_{I_{i} J_{i}}^{\mu_{i}}\left(x_{i}\right)=\left(\Psi_{\beta_{i} J_{i}} \star \bar{\Psi}_{\alpha_{i} I_{i}}\right)\left(x_{i}\right)\left(\bar{\gamma}^{\mu_{i}} \gamma_{5}\right)_{\alpha_{i} \beta_{i}} .
$$

Here the symbol $\langle\mathcal{O}\rangle_{0}^{\text {conn }}$ stands for the connected component of the correlation function $\langle\mathcal{O}\rangle_{0}$ defined by

$$
\langle\mathcal{O}\rangle_{0}=\int[d \bar{\Psi}][d \Psi] \mathcal{O} \exp \left(i \int d^{2 \omega_{x}} \bar{\Psi}_{I} \not \partial \Psi_{I}\right) .
$$

Note that eqs. (3.8) and (3.10) define $\Gamma_{I_{1} J_{1} \ldots I_{n} J_{n}}^{\mu_{1} \ldots \mu_{n}}\left(x_{1}, \ldots, x_{n}\right)$ as the result of applying Wick's theorem to $\mathcal{J}_{I_{1} J_{1}}^{\mu_{1}}\left(x_{1}\right) \ldots \mathcal{J}_{I_{n} J_{n}}^{\mu_{n}}\left(x_{n}\right)$ with regard to the contraction

$$
\left\langle\Psi_{\beta J}(y) \bar{\Psi}_{\alpha I}(x)\right\rangle_{0}=\delta_{J I} \int \frac{d^{2 \omega} q}{(2 \pi)^{2 \omega}} e^{-i q(y-x)} \frac{i \not_{\beta \alpha}}{q^{2}+i 0^{+}} .
$$

It is not difficult to see that in eq. (3.8) there are $(n-1)$ ! different contractions and that, upon combination with the $V^{\prime}$ s in eq. (3.7), they all yield the same contribution. The regularized effective action then takes the form

$$
i \Gamma^{\mathrm{reg}}[V]=-\sum_{n=1}^{\infty} \frac{(-1)^{n}}{n} \int d^{2 \omega} x_{1} \ldots \int d^{2 \omega} x_{n} \operatorname{Tr}\left[V_{\mu_{1}}\left(x_{1}\right) \ldots V_{\mu_{n}}\left(x_{n}\right)\right] \Gamma^{\mu_{1} \ldots \mu_{n}}\left(x_{1}, \ldots, x_{n}\right),
$$


where

$$
\operatorname{Tr}\left[V_{\mu_{1}}\left(x_{1}\right) \ldots V_{\mu_{n}}\left(x_{n}\right)\right]=V_{\mu_{1} I_{1} I_{2}}\left(x_{1}\right) V_{\mu_{2} I_{2} I_{3}}\left(x_{2}\right) \ldots V_{\mu_{n-1} I_{n-1} I_{n}}\left(x_{n-1}\right) V_{\mu_{n} I_{n} I_{1}}\left(x_{n}\right),
$$

the 1PI Green function $\Gamma_{\mu_{1} \ldots \mu_{n}}\left(x_{1}, \ldots, x_{n}\right)$ reads

$$
\begin{aligned}
\Gamma_{\mu_{1} \ldots \mu_{n}}\left(x_{1}, \ldots, x_{n}\right)= & i^{n} \int \prod_{i=1}^{n} \frac{d^{2 \omega} p_{i}}{(2 \pi)^{2 \omega}}(2 \pi)^{2 \omega} \delta\left(p_{1}+\cdots+p_{n}\right) e^{i \sum_{i=1}^{n} p_{i} x_{i}} e^{-\frac{i}{2} \sum_{1 \leq i<j<n} \theta^{\alpha \beta} p_{i \alpha} p_{j \beta}} \times \\
& \times \int \frac{d^{2 \omega} q}{(2 \pi)^{2 \omega}} \frac{\operatorname{tr}\left[\left(\not q+\not p_{1}\right) \bar{\gamma}^{\mu_{1}} \mathrm{P}_{-} \not \bar{\gamma}^{\mu_{2}} \mathrm{P}_{-}\left(\not q-\not p_{2}\right) \ldots\left(\not q-\sum_{i=2}^{n-1} \not \not_{i}\right) \bar{\gamma}^{\mu_{n}} \mathrm{P}_{-}\right]}{\left(q+p_{1}\right)^{2} q^{2}\left(q-p_{2}\right)^{2} \cdots\left(q-\sum_{i=2}^{n-1} p_{i}\right)^{2}}
\end{aligned}
$$

and the symbol tr denotes trace over Dirac matrices. For completeness we present very briefly an alternative derivation of (3.12). Integrating over $[d \bar{\Psi}]$ and $[d \Psi]$ in eq. (3.5) and using eq. (3.6), we obtain

$$
i \Gamma^{\mathrm{reg}}[V]=\operatorname{Tr} \ln \left[1+(\not \partial)^{-1} \bar{\gamma}^{\mu} V_{\mu} \mathrm{P}_{-\star}\right]=-\sum_{n=1}^{n} \frac{(-1)^{n}}{n} \operatorname{Tr}\left[(\not \partial)^{-1} \bar{\gamma}^{\mu} V_{\mu} \mathrm{P}_{-\star}\right]^{n},
$$

where Tr is to be interpreted as $\int d^{2 \omega} x$ for the continuous indices of the operator on which $\operatorname{Tr}$ acts and $(\not \partial)^{-1}$ has matrix elements $\left\langle y\left|(\not \partial)^{-1}\right| x\right\rangle$ given by the right-hand-side of eq. (3.11). Clearly the right-hand side of eq. (3.14) has a neat diagrammatic representation which readily leads to eq. (3.12).

We stress the fact that the noncommutative field $V_{\mu}(x)$ in eqs. (3.12) and (3.14) is a mere spectator in the sense that these equations hold whatever the algebra on which $V_{\mu}(x)$ takes values be, provided the operation $\operatorname{Tr}$ make sense. Eqs. (3.12) and (3.14) are thus valid for noncommutative $\mathrm{U}(N)$, simple, semisimple and non-semisimple gauge groups. One then expects that for nonsemisimple gauge groups a renormalized effective action $\Gamma^{\mathrm{ren}}[V]$ can be defined so that the noncommutative gauge anomaly has the same form as for noncommutative $\mathrm{U}(N)$ group, i.e. such that

$$
s_{\star} \Gamma^{\mathrm{ren}}[V]=\mathcal{A}_{\star},
$$

with

$$
\mathcal{A}_{\star}=-\frac{i}{24 \pi^{2}} \int d^{4} x \epsilon^{\mu_{1} \mu_{2} \mu_{3} \mu_{4}} \operatorname{Tr} \Lambda \star \partial_{\mu_{1}}\left(V_{\mu_{2}} \star \partial_{\mu_{3}} V_{\mu_{4}}+\frac{1}{2} V_{\mu_{2}} \star V_{\mu_{3}} \star V_{\mu_{4}}\right) .
$$

In the remainder of the section we prove that is indeed so.

To demonstrate eqs. (3.15) and (3.16) we proceed as follows. Since the integral over $d^{2 \omega} q$ in eq. (3.12) does not involve any nonplanar factor $e^{i q_{\alpha} \theta^{\alpha \beta} p_{i \beta}}$, the effective action in eq. (3.12) is given by a sum over dimensionally regularized planar diagrams. Hence, the Quantum action principle [22] holds for this effective action and the following equation is valid

$$
s_{\star} \Gamma^{\mathrm{reg}}[V]=\hat{\Delta} \cdot \Gamma^{\mathrm{reg}}[V] .
$$


Here $\hat{\Delta} \cdot \Gamma^{\text {reg }}[V]$ is the insertion in $\Gamma^{\text {reg }}[V]$ of the evanescent operator $\hat{\Delta}$ defined by

$$
\hat{\Delta}=s_{\star} S_{\mathrm{nc}}^{\mathrm{reg}}=\int d^{2 \omega} x\left[\bar{\Psi}_{I} \star \Lambda_{I J} \star i \hat{\not} \mathrm{P}_{+} \Psi_{J}-\bar{\Psi}_{I} \star i \hat{\not}\left(\Lambda_{I J} \star \mathrm{P}_{-} \Psi_{J}\right)\right] .
$$

Substituting this in eq. (3.17), we obtain for its right-hand side

$$
\begin{aligned}
\hat{\Delta} \cdot \Gamma^{\mathrm{reg}}[V]= & -\sum_{n=1}^{\infty}(-1)^{n} \int d^{2 \omega} x \int d^{2 \omega} x_{1} \ldots \int d^{2 \omega} x_{n} \times \\
& \times \operatorname{Tr}\left[\Lambda(x) V_{\mu_{1}}\left(x_{1}\right) \ldots V_{\mu_{n}}\left(x_{n}\right)\right] \Gamma^{\mu_{1} \ldots \mu_{n}}\left(x, x_{1}, \ldots, x_{n} \mid \hat{\Delta}\right),
\end{aligned}
$$

where

$$
\begin{aligned}
\Gamma^{\mu_{1} \ldots \mu_{n}}\left(x, x_{1}, \ldots, x_{n} \mid \hat{\Delta}\right)= & i^{n+1} \int \frac{d^{2 \omega} p}{(2 \pi)^{2 \omega}} \int \prod_{i=1}^{n} \frac{d^{2 \omega} p_{i}}{(2 \pi)^{2 \omega}} e^{i\left(p x+\sum_{i=1}^{n} p_{i} x_{i}\right)} \times \\
& \times e^{-\frac{i}{2} \sum_{1 \leq i<j<n} \theta^{\alpha \beta} p_{i \alpha} p_{j \beta}}(2 \pi)^{2 \omega} \delta\left(p+p_{1}+\cdots p_{n}\right) \times \\
& \times \Gamma^{\mu_{1} \ldots \mu_{n}}\left(p, p_{1}, \ldots, p_{n} \mid \hat{\Delta}\right),
\end{aligned}
$$

and the 1PI Green function $\Gamma^{\mu_{1} \ldots \mu_{n}}\left(p, p_{1}, \ldots, p_{n} \mid \hat{\Delta}\right)$ with the insertion reads

$$
\begin{aligned}
\Gamma^{\mu_{1} \ldots \mu_{n}}\left(p, p_{1}, \ldots, p_{n} \mid \hat{\Delta}\right)= & \int \frac{d^{2 \omega} q}{(2 \pi)^{2 \omega}} \frac{1}{q^{2}\left(q-p_{1}\right)^{2}\left(q-p_{1}-p_{2}\right)^{2} \ldots\left(q-\sum_{i=1}^{n} p_{i}\right)^{2}} \times \\
& \times \operatorname{tr}\left[\hat{q} \mathrm{P}_{+}-\left(\hat{\not q}-\sum_{i=1}^{n} \hat{\not p}_{i}\right) \mathrm{P}_{-}\right] \times \\
& \times q \bar{\gamma}^{\mu_{1}} \mathrm{P}_{-}\left(\not q-\not \not_{1}\right) \bar{\gamma}^{\mu_{2}} \mathrm{P}_{-}\left(\not q-\not p_{1}-\not p_{2}\right) \ldots \bar{\gamma}^{\mu_{n}} \mathrm{P}_{-}\left(\not q-\sum_{i=1}^{n} \not p_{i}\right) .
\end{aligned}
$$

As before, tr denotes trace over Dirac matrices. For $n \geq 5$ the integral in eq. (3.20) is UV finite by power counting at $2 \omega=4$. Hence,

$$
\Gamma^{\mu_{1} \ldots \mu_{n}}\left(p, p_{1}, \ldots, p_{n} \mid \hat{\Delta}\right)=O(\varepsilon) \quad n \geq 5,
$$

where $\varepsilon=\omega-2$. As concerns $n \leq 4$, using the results in appendix A it is straightforward to compute the contribution $\Gamma_{\text {eps }}^{\mu_{1} \ldots \mu_{n}}\left(p, p_{1}, \ldots, p_{n} \mid \hat{\Delta}\right)$ to $\Gamma^{\mu_{1} \ldots \mu_{n}}\left(p, p_{1}, \ldots, p_{n} \mid \hat{\Delta}\right)$ involving $\epsilon^{\mu_{1} \mu_{2} \mu_{3} \mu_{4}}$. After some calculations, we obtain

$$
\begin{aligned}
\Gamma_{\mathrm{eps}}^{\mu_{1}}\left(p, p_{1} \mid \hat{\Delta}\right) & =0 \\
\Gamma_{\mathrm{eps}}^{\mu_{1} \mu_{2}}\left(p, p_{1}, p_{2} \mid \hat{\Delta}\right) & =\frac{1}{24 \pi^{2}} \epsilon^{\rho \mu_{1} \sigma \mu_{2}} p_{1 \rho} p_{2 \sigma}+O(\varepsilon) \\
\Gamma_{\mathrm{eps}}^{\mu_{1} \mu_{2} \mu_{3}}\left(p, p_{1}, p_{2}, p_{3} \mid \hat{\Delta}\right) & =-\frac{1}{2} \frac{1}{24 \pi^{2}} \epsilon^{\rho \mu_{1} \mu_{2} \mu_{3}}\left(p_{1}+p_{2}+p_{3}\right)_{\rho}+O(\varepsilon) \\
\Gamma_{\mathrm{eps}}^{\mu_{1} \mu_{2} \mu_{3} \mu_{4}}\left(p, p_{1}, p_{2}, p_{3}, p_{4} \mid \hat{\Delta}\right) & =O(\varepsilon) .
\end{aligned}
$$

Substituting eqs. (3.21) and (3.22) in eq. (3.19), and the result so obtained in eq. (3.18), we have that the contribution to the right-hand side of eq. (3.17) which contains $\epsilon^{\mu_{1} \mu_{2} \mu_{3} \mu_{4}}$ reads

$$
\left.\hat{\Delta} \cdot \Gamma^{\mathrm{reg}}[V]\right|_{\mathrm{eps}}=\mathcal{A}_{\star}^{\mathrm{reg}}
$$


where

$$
\mathcal{A}_{\star}^{\mathrm{reg}}=-\frac{i}{24 \pi^{2}} \int d^{2 \omega} x \epsilon^{\mu_{1} \mu_{2} \mu_{3} \mu_{4}} \operatorname{Tr} \Lambda \star \partial_{\mu_{1}}\left(V_{\mu_{2}} \star \partial_{\mu_{3}} V_{\mu_{4}}+\frac{1}{2} V_{\mu_{2}} \star V_{\mu_{3}} \star V_{\mu_{4}}\right)+O(\varepsilon) .
$$

Hence, if $\Gamma_{\text {eps }}^{\mathrm{reg}}[V]$ denotes the contribution to the regularized effective action $\Gamma^{\mathrm{reg}}[V]$ involving $\epsilon^{\mu_{1} \mu_{2} \mu_{3} \mu_{4}}$, eqs. (3.17) and (3.23) imply

$$
s_{\star} \Gamma_{\mathrm{eps}}^{\mathrm{reg}}[V]=\left.\hat{\Delta} \cdot \Gamma^{\mathrm{reg}}[V]\right|_{\mathrm{eps}}=\mathcal{A}_{\star}^{\mathrm{reg}} .
$$

It is not difficult to show that the pole part of $\Gamma^{\mathrm{reg}}[V]$ at $\varepsilon=0$ does not depend on $\epsilon^{\mu_{1} \mu_{2} \mu_{3} \mu_{4}}$. This, together with the observation that any vector-like contribution to the regularized effective action - i.e. not involving $\epsilon^{\mu_{1} \mu_{2} \mu_{3} \mu_{4}}$ - can be regularized in a gauge invariant way within the framework of dimensional regularization, implies that it is always possible to define a renormalized effective action

$$
\Gamma^{\mathrm{ren}}[V]=\Gamma_{\mathrm{vec}-\text { like }}^{\mathrm{ren}}[V]+\Gamma_{\mathrm{eps}}^{\mathrm{ren}}[V]
$$

such that

$$
s_{\star} \Gamma_{\text {vec-like }}^{\text {ren }}[V]=0
$$

and

$$
s_{\star} \Gamma_{\mathrm{eps}}^{\mathrm{ren}}[V]=\lim _{\varepsilon \rightarrow 0} \mathcal{A}_{\star}^{\mathrm{reg})}=\mathcal{A}_{\star},
$$

with $\mathcal{A}_{\star}$ as in eq. (3.16). Hence eqs. (3.15) and (3.16) follow.

Using finally that $s_{\star} V_{\mu}=s V_{\mu}$ and that $V_{\mu}$ is a function of $v_{\mu}$ and $\theta^{\mu \nu}$ we conclude that

$$
s \Gamma^{\mathrm{ren}}[v ; \theta]=\mathcal{A}_{\star} .
$$

This equation gives a simple expression for the anomaly if written in terms of the noncommutative fields $V_{\mu}$ and $\lambda$. In fact, $\mathcal{A}_{\mathrm{nc}}^{\text {Bardeen }}$ in eq. (3.16) is nothing but the noncommutative counterpart of Bardeen's ordinary anomaly. However, in terms of the fields $v_{\mu}$ and $\lambda$, the anomaly is a complicated power series in $\theta^{\mu \nu}$ with coefficients depending on such fields. The first term of such series is the standard Bardeen anomaly $\mathcal{A}^{\text {Bardeen }}$ of ordinary spacetime,

$$
\begin{aligned}
\mathcal{A}_{\star} & =\mathcal{A}^{\text {Bardeen }}+O(\theta) \\
\mathcal{A}^{\text {Bardeen }}=\left.\mathcal{A}_{\star}\right|_{\theta=0} & =-\frac{i}{24 \pi^{2}} \int d^{4} x \epsilon^{\mu_{1} \mu_{2} \mu_{3} \mu_{4}} \operatorname{Tr} \lambda \partial_{\mu_{1}}\left(v_{\mu_{2}} \partial_{\mu_{3}} v_{\mu_{4}}+\frac{1}{2} v_{\mu_{2}} v_{\mu_{3}} v_{\mu_{4}}\right) .
\end{aligned}
$$

\section{BRS triviality of $\theta$-dependent contributions}

The functional $\mathcal{A}_{\star}$ in eqs. (3.15) and (3.16) has been found by explicitly computing to all orders in $\theta^{\mu \nu}$ the $\epsilon^{\mu_{1} \mu_{2} \mu_{3} \mu_{4}}$ part of the one-loop radiative corrections to all the 1PI Green functions of the field $V_{\mu}$. As is well known, only radiative corrections which are cohomologically nontrivial with respect to the ordinary chiral BRS operator $s$, that is to say, that can not be written as the $s$ of something, yield a true anomalous contribution. To find the true anomaly, we must therefore identify in $\mathcal{A}_{\star}$ the cohomologically nontrivial contributions with respect to $s$. This we do next. 
If in sections 2 and 3 we take as noncommutativity matrix $t \theta^{\mu \nu}$, with $t$ a real parameter, we end up with a noncommutative BRS chiral operator $s_{\star}^{(t \theta)}$ and an anomaly $\mathcal{A}_{\star}^{(t \theta)}$ whose expressions are obtained from those in sections 2 and 3 by replacing $\theta^{\mu \nu}$ with $t \theta^{\mu \nu}$. Note that the dependence on $t$ of $s_{\star}^{(t \theta)}$ is only through the Moyal product, which now is with respect to $t \theta^{\mu \nu}$, but that no explicit $t$-dependence is introduced (see ref. [21]). In appendix B we prove that the logarithmic differential with respect to $t$ of $\mathcal{A}_{\star}^{(t \theta)}$ is $s_{\star}^{(t \theta)}$ trivial, or in other words, that there exists a functional $\mathcal{B}\left[V^{(t \theta)}, t \theta\right]$ such that

$$
t \frac{d}{d t} \mathcal{A}_{\star}^{(t \theta)}=s_{\star}^{(t \theta)} \mathcal{B}\left[V^{(t \theta)}, t \theta\right] .
$$

Let us remark that we use the logarithmic derivative $t \frac{d}{d t}$, and not the ordinary derivative $d / d t$ as in refs. [23, 21], to be able to write everything in terms of the noncommutativity matrix $t \theta^{\mu \nu}$ and to avoid having to use both $\theta^{\mu \nu}$ and $t \theta^{\mu \nu}$. Integrating eq. (4.1) over $t$ from 0 to 1 and using that - by definition of the Seiberg-Witten map $-s_{\star}^{(t \theta)} V_{\mu}^{(t \theta)}=s V_{\mu}^{(t \theta)}[v, t \theta]$, we have

$$
\int_{0}^{1} d t \frac{d \mathcal{A}_{\star}^{(t \theta)}}{d t}=\int_{0}^{1} \frac{d t}{t} s_{\star}^{(t \theta)} \mathcal{B}\left[V^{(t \theta)}, t \theta\right]=\int_{0}^{1} \frac{d t}{t} s \mathcal{B}\left[V^{(t \theta)}[v, t \theta], t \theta\right] .
$$

Recalling now that $\mathcal{A}_{\star}^{(t \theta)}=\mathcal{A}_{\star}$ if $t=1$ and $\mathcal{A}_{\star}^{(t \theta)}=\mathcal{A}^{\text {Bardeen }}$ if $t=0$, and noting that the ordinary BRS chiral operator $s$ does not depend on $t$, we obtain

$$
\mathcal{A}_{\star}=\mathcal{A}^{\text {Bardeen }}-s \int_{0}^{1} \frac{d t}{t} \mathcal{B}_{\star}\left[V^{(t \theta)}[v, t \theta], t \theta\right] .
$$

Hence the functional $\mathcal{A}_{\star}$ found in section 3 consists of two contributions: the standard Bardeen anomaly $\mathcal{A}^{\text {Bardeen }}$ of commutative spacetime, and a contribution — given by the second term in eq. (4.2) - which is cohomologically trivial with respect to the ordinary chiral BRS operator $s$. Comparing with eq. (3.26), we conclude that all contributions to $\mathcal{A}_{\star}$ of order one or higher in $\theta^{\mu \nu}$ are cohomologically trivial, hence harmless, since they can be absorbed by adding finite counterterms to the renormalized effective action. Indeed, consider a new renormalized effective action $\Gamma^{\prime \prime \text { ren }}[v ; \theta]$ defined by

$$
\Gamma^{\prime \operatorname{ren}}[v ; \theta]=\Gamma^{\mathrm{ren}}[v ; \theta]-\int_{0}^{1} \frac{d t}{t} \mathcal{B}\left[V^{(t \theta)}[v, t \theta], t \theta\right] .
$$

According to our discussion above, it follows that

$$
s \Gamma^{\prime \text { ren }}[v ; \theta]=\mathcal{A}^{\text {Bardeen }} .
$$

We thus conclude that the anomaly is $\theta^{\mu \nu}$-independent and has Bardeen's form.

\section{Conclusion}

In this paper we have calculated the chiral one-loop anomaly in 4-dimensional noncommutative gauge theories with arbitrary compact gauge group defined through the Seiberg-Witten map. Our main result is that for all these theories the chiral anomaly is the same as for their 
commutative counterparts. Hence any noncommutative chiral gauge theory of this type is anomaly free to one-loop order if, and only if, its ordinary counterpart is. This implies in particular that the anomaly cancellation conditions for the noncommutative standard model [6] and the noncommutative $\mathrm{SU}(5)$ and $\mathrm{SO}(10)$ models [13] are the same as for the ordinary ones [27]. We would like to emphasize that we have not found anomaly candidates but actually computed the anomaly, since we have calculated the relevant Feynman diagrams that produce the anomaly.

There is one key ingredient in our proof, namely that counterterms with mass dimension greater than four should be allowed in the renormalized effective action. This is necessary to cancel radiative corrections which, on the one hand, do not satisfy the equation $s \Gamma^{\mathrm{ren}}=0$ but, on the other, are cohomologically trivial with respect to $s$. This indicates that the proper framework for these theories is the effective field theory formalism, a proposal that has already been made by a number of authors [13, 17, 18. If one insists on power-counting renormalizability, then the "safe" representations and the safe "groups" of ordinary gauge theories [24] are totally unsafe for noncommutative gauge theories, since they lead to anomalous theories [18].

\section{Acknowledgments}

CPM and FRR are grateful to CICyT, Spain for partial support through grant No. BFM2002-00950.

\section{A. Useful integrals}

To obtain the $\epsilon^{\mu_{1} \mu_{2} \mu_{3} \mu_{4}}$ contribution to the $n$-point functions $\Gamma^{\mu_{1} \ldots \mu_{n}}\left(p, p_{1}, \ldots, p_{n} \mid \hat{\Delta}\right)$ with one evanescent insertion $\hat{\Delta}$ given in eqs. (3.22) the following integrals are needed:

$$
\begin{gathered}
\int \frac{d^{2 \omega} q}{(2 \pi)^{2 \omega}} \frac{\hat{q}^{2}}{q^{2}\left(q-q_{1}\right)^{2}\left(q-p_{2}\right)^{2}}=-\frac{1}{2} \frac{i}{16 \pi^{2}}+O(\varepsilon) \\
\int \frac{d^{2 \omega} q}{(2 \pi)^{2 \omega}} \frac{\hat{q}^{2} \bar{q}_{\mu}}{q^{2}\left(q-q_{1}\right)^{2}\left(q-q_{2}\right)^{2}}=-\frac{1}{6} \frac{i}{16 \pi^{2}}\left(\bar{q}_{1}+\bar{q}_{2}\right)_{\mu}+O(\varepsilon) \\
\int \frac{d^{2 \omega} q}{(2 \pi)^{2 \omega}} \frac{1}{q^{2}\left(q-q_{1}\right)^{2}\left(q-q_{2}\right)^{2}\left(q-q_{3}\right)^{2}}=-\frac{i}{12} \frac{\hat{q}_{\mu_{1}} \bar{q}_{\mu_{2}}}{16 \pi^{2}} \bar{g}_{\mu_{1} \mu_{2}}+O(\varepsilon) \\
\int \frac{d^{2 \omega} q}{(2 \pi)^{2 \omega}} \frac{\hat{q}^{2} \bar{q}_{\mu_{1}} \bar{q}_{\mu_{2}} \bar{q}_{\mu_{3}}}{q^{2}\left(q-q_{1}\right)^{2}\left(q-q_{2}\right)^{2}\left(q-q_{3}\right)^{2}}= \\
=-\frac{1}{48} \frac{i}{16 \pi^{2}} \sum_{i=1}^{3}\left(\bar{g}_{\mu_{1} \mu_{2}} \bar{q}_{i \mu_{3}}+\bar{g}_{\mu_{1} \mu_{3}} \bar{q}_{i \mu_{2}}+\bar{g}_{\mu_{2} \mu_{3}} \bar{q}_{i \mu_{1}}\right)+O(\varepsilon) \\
\int \frac{d^{2 \omega} q}{(2 \pi)^{2 \omega}} \frac{1}{\hat{q}^{2}\left(q-\bar{q}^{2} \bar{q}_{\mu_{1}} \bar{q}_{\mu_{2}}\left(q-q_{2}\right)^{2}\left(q-q_{3}\right)^{2}\left(q-q_{4}\right)^{2}\right.}=-\frac{1}{16} \frac{i}{16 \pi^{2}} \bar{g}_{\mu_{1} \mu_{2}}+O(\varepsilon) .
\end{gathered}
$$

Here $\varepsilon=\omega-2$. 


\section{B. Proof of eq. 4.1}

In what follows we will use $\omega^{\alpha \beta}$ for $t \theta^{\alpha \beta}$, denote the Moyal product with respect to $\omega^{\alpha \beta}$ by $\star$ and write a small circle ${ }^{\circ}$ for the logarithmic differential with respect to $t$, i.e.

$$
\omega^{\alpha \beta}=t \theta^{\alpha \beta} \quad \star=\star_{\omega} \quad \stackrel{\circ}{\mathcal{F}}=t \frac{d \mathcal{F}}{d t} .
$$

The functional $\mathcal{A}_{\star}^{(t \theta)}$, which in this notation we write as $\mathcal{A}_{\star}$, has a piece of order zero in $\omega^{\alpha \beta}$ given by $\mathcal{A}^{\text {Bardeen }}$ in eq. (3.27) and a piece that collects all the higher order terms in $\omega^{\alpha \beta}$ and which precisely gives the contributions to $\stackrel{\circ}{\mathcal{A}}_{\star}$. We want to prove eq. (4.1), which now takes the form

$$
\stackrel{\circ}{\mathcal{A}}_{\star}=s_{\star} \mathcal{B}
$$

Using

$$
f \stackrel{\circ}{\star} g=\frac{1}{2} \omega^{\alpha \beta} \partial_{\alpha} f \star \partial_{\beta} g
$$

and [23]

$$
\stackrel{\circ}{V}_{\mu}=-\frac{i}{4} \omega^{\alpha \beta}\left\{V_{\alpha}, F_{\beta \mu}+\partial_{\beta} V_{\mu}\right\}_{\star} \quad \stackrel{\circ}{\Lambda}=\frac{i}{4} \omega^{\alpha \beta}\left\{\partial_{\alpha} \Lambda, V_{\beta}\right\}_{\star},
$$

the functional $\stackrel{\circ}{\mathcal{A}_{\star}}$ can be expanded as a sum

$$
\stackrel{\circ}{\mathcal{A}}_{\star}=\stackrel{\circ}{\mathcal{A}}_{\star, 3}+\stackrel{\circ}{\mathcal{A}}_{\star, 4}+\stackrel{\circ}{\mathcal{A}}_{\star, 5}+\stackrel{\circ}{\mathcal{A}}_{\star, 6},
$$

where $\stackrel{\circ}{\mathcal{A}}_{\star, n}$ collects all contributions in $\stackrel{\circ}{\mathcal{A}}_{\star}$ of degree $n$ in the fields $\Lambda$ and $V_{\mu}$ (see below for their explicit expressions). In turn, the noncommutative chiral BRS operator $s_{\star}$ can be written as the sum

$$
s_{\star}=s_{\star, 0}+s_{\star, 1}
$$

of two operators $s_{\star, 0}$ and $s_{\star, 1}$ whose action on the fields $\Lambda$ and $V_{\mu}$ is given by

$$
\begin{array}{ll}
s_{\star, 0} V_{\mu}=\partial_{\mu} \Lambda & s_{\star, 0} \Lambda=0 \\
s_{\star, 1} V_{\mu}=\left[V_{\mu}, \Lambda\right]_{\star} & s_{\star, 1} \Lambda=-\Lambda \star \Lambda .
\end{array}
$$

These two operators satisfy

$$
s_{\star, 0}^{2}=0 \quad s_{\star, 0} s_{\star, 1}+s_{\star, 1} s_{\star, 0}=0
$$

and have the important property that $s_{\star, 0}$ preserves the degree in the fields and $s_{\star, 1}$ increases it by one. From eqs. (B.5) and (B.6) it follows that to prove eq. (B.2) it is sufficient to take for $\mathcal{B}$ an expansion

$$
\mathcal{B}=\mathcal{B}_{3}+\mathcal{B}_{4}+\mathcal{B}_{5}+\mathcal{B}_{6}
$$

in the number of fields and show that

$$
\begin{aligned}
& \stackrel{\circ}{\mathcal{A}}_{\star, 3}=s_{\star, 0} \mathcal{B}_{3} \\
& \stackrel{\circ}{\mathcal{A}}_{\star, 4}-s_{\star, 1} \mathcal{B}_{3}=s_{\star, 0} \mathcal{B}_{4}
\end{aligned}
$$




$$
\begin{aligned}
{\stackrel{\circ}{\mathcal{A}_{\star}, 5}-s_{\star, 1}} \mathcal{B}_{4} & =s_{\star, 0} \mathcal{B}_{5} \\
\stackrel{\circ}{\mathcal{A}}_{\star, 6}-s_{\star, 1} \mathcal{B}_{5} & =s_{\star, 0} \mathcal{B}_{6} \\
s_{\star, 1} \mathcal{B}_{6} & =0 .
\end{aligned}
$$

Hence, to prove (B.2) all we have to do is finding functionals $\mathcal{B}_{3}, \mathcal{B}_{4}, \mathcal{B}_{5}$ and $\mathcal{B}_{6}$ satisfying the ladder equations. To do this it is convenient to use differential forms, so let us write eqs. (B.7) and (B.8) in terms of differential forms. Recalling that $V=V_{\mu} d x^{\mu}$ and using $\left\{d x^{\mu}, s_{\star, 0}\right\}=\left\{d x^{\mu}, s_{\star, 1}\right\}=\left\{\Lambda, d x^{\mu}\right\}=0$, we have

$$
\begin{array}{ll}
s_{\star, 0} V=-d \Lambda & s_{\star, 0} \Lambda=0 \\
s_{\star, 1} V=-\{V, \Lambda\}_{\star} & s_{\star, 1} \Lambda=-\Lambda \star \Lambda .
\end{array}
$$

\section{B.1 Computation of $\mathcal{B}_{3}$ and $\mathcal{B}_{4}$}

Taking the logarithmic differential with respect to $t$ of $\mathcal{A}_{\star}$ and using eqs. (B.3) and (B.4), it is straightforward to see that

$$
\stackrel{\circ}{\mathcal{A}}_{\star, 3}=-\frac{i}{24 \pi^{2}} \int \frac{i}{2} \omega^{\alpha \beta} \operatorname{Tr} \partial_{\alpha} \Lambda \star \partial_{\beta} d V \star d V .
$$

It is clear that

$$
\mathcal{B}_{3}=-\frac{i}{24 \pi^{2}} \int \frac{i}{2} \omega^{\alpha \beta} \operatorname{Tr}\left[x V_{\alpha} \star \partial_{\beta} d V \star d V-(1-x) V_{\alpha} \star d V \star \partial_{\beta} d V\right],
$$

with $x$ an arbitrary parameter, solves eq. (B.9). Indeed, acting with $s_{\star, 0}$ on $\mathcal{B}_{3}$, integrating by parts the derivative $\partial_{\beta}$ in the second term in eq. (B.16) and neglecting the integral of a divergence, we recover $\stackrel{\circ}{*}_{\star 3}$. Note that eq. (B.16) provides a one-parameter family of solutions for $\mathcal{B}_{3}$. Furthermore, to $\mathcal{B}_{3}$ we can also add a term

$$
\int \omega^{\alpha \beta} \operatorname{Tr} \partial_{\alpha} V_{\beta} \star d V \star d V
$$

with arbitrary coefficient, since $s_{\star, 0}$ acting on it vanishes.

Let us move now on to eq. (B.10). We first calculate $\stackrel{\circ}{\mathcal{A}}_{\star, 4}$ and $s_{\star, 1} \mathcal{B}_{3}$. Acting with $t \frac{d}{d t}$ on $\mathcal{A}_{\star}$, noting eqs. (B.3) and (B.4), retaining terms of order four in the fields, using the cyclic property of the trace $\operatorname{Tr}$ and of the integral of a Moyal product of functions to push the ghost field $\Lambda$ to the far left, and integrating by parts whatever partial and/or exterior derivatives act on $\Lambda$, we obtain after some lengthy algebra that

$$
\begin{aligned}
\stackrel{\circ}{\mathcal{A}} \star 4_{\star}=- & \frac{i}{24 \pi^{2}} \int \frac{i}{4} \omega^{\alpha \beta} \operatorname{Tr} \Lambda \star \star \\
\times & {\left[V_{\alpha} \star d V \star \partial_{\beta} d V-V_{\alpha} \star \partial_{\beta} d V \star d V+V \star \partial_{\alpha} V \star \partial_{\beta} d V-V \star \partial_{\alpha} d V \star \partial_{\beta} V-\right.} \\
& \quad-\partial_{\alpha} V_{\beta} \star d V \star d V-d V \star d V \star \partial_{\alpha} V_{\beta}-2 \partial_{\alpha} V \star d V_{\beta} \star d V+2 d V_{\alpha} \star d V_{\beta} \star d V- \\
& \quad-2 d V_{\alpha} \star \partial_{\beta} V \star d V-2 d V \star \partial_{a} V \star d V_{\beta}+2 d V \star d V_{\alpha} \star d V_{\beta}-2 d V \star d V_{\alpha} \star \partial_{\beta} V+ \\
& +d V \star \partial_{\alpha} V \star \partial_{\beta} V-\partial_{\alpha} V \star \partial_{\beta} V \star d V-\partial_{\alpha} V \star d V \star \partial_{\beta} V-\partial_{\alpha} d V \star d V \star V_{\beta}+
\end{aligned}
$$




\begin{tabular}{||c|c||c|c||c|c|}
\hline$a_{\alpha \beta}^{1}$ & $d V \star \partial_{\alpha} V \star \partial_{\beta} V$ & $a_{\alpha \beta}^{14}$ & $\partial_{\alpha} V \star d V \star d V_{\beta}$ & $a_{\alpha \beta}^{27}$ & $V \star d V_{\alpha} \star \partial_{\beta} d V$ \\
$a_{\alpha \beta}^{2}$ & $\partial_{\alpha} V \star d V \star \partial_{\beta} V$ & $a_{\alpha \beta}^{15}$ & $\partial_{\alpha} V \star d V_{\beta} \star d V$ & $a_{\alpha \beta}^{28}$ & $\partial_{\alpha} d V \star d V_{\beta} \star V$ \\
$a_{\alpha \beta}^{3}$ & $\partial_{\alpha} V \star \partial_{\beta} V \star d V$ & $a_{\alpha \beta}^{16}$ & $d V \star d V_{\alpha} \star \partial_{\beta} V$ & $a_{\alpha \beta}^{29}$ & $d V_{\alpha} \star \partial_{\beta} d V \star V$ \\
$a_{\alpha \beta}^{4}$ & $\partial_{\alpha} d V \star V \star \partial_{\beta} V$ & $a_{\alpha \beta}^{17}$ & $d V_{\alpha} \star d V \star \partial_{\beta} V$ & $a_{\alpha \beta}^{30}$ & $d V_{\alpha} \star V \star \partial_{\beta} d V$ \\
$a_{\alpha \beta}^{5}$ & $V \star \partial_{\alpha} d V \star \partial_{\beta} V$ & $a_{\alpha \beta}^{18}$ & $d V_{\alpha} \star \partial_{\beta} V \star d V$ & $a_{\alpha \beta}^{31}$ & $\partial_{\alpha} d V_{\beta} \star d V \star V$ \\
$a_{\alpha \beta}^{6}$ & $V \star \partial_{\alpha} V \star \partial_{\beta} d V$ & $a_{\alpha \beta}^{19}$ & $\partial_{\alpha} d V \star d V \star V_{\beta}$ & $a_{\alpha \beta}^{32}$ & $d V \star \partial_{\alpha} d V_{\beta} \star V$ \\
$a_{\alpha \beta}^{7}$ & $\partial_{\alpha} d V \star \partial_{\beta} V \star V$ & $a_{\alpha \beta}^{20}$ & $d V \star \partial_{\alpha} d V \star V_{\beta}$ & $a_{\alpha \beta}^{33}$ & $d V \star V \star \partial_{\alpha} d V_{\beta}$ \\
$a_{\alpha \beta}^{8}$ & $\partial_{\alpha} V \star \partial_{\beta} d V \star V$ & $a_{\alpha \beta}^{21}$ & $d V \star V_{\alpha} \star \partial_{\beta} d V$ & $a_{\alpha \beta}^{34}$ & $\partial_{\alpha} d V_{\beta} \star V \star d V$ \\
$a_{\alpha \beta}^{9}$ & $\partial_{\alpha} V \star V \star \partial_{\beta} d V$ & $a_{\alpha \beta}^{22}$ & $\partial_{\alpha} d V \star V_{\beta} \star d V$ & $a_{\alpha \beta}^{35}$ & $V \star \partial_{\alpha} d V_{\beta} \star d V$ \\
$a_{\alpha \beta}^{10}$ & $d V \star d V \star \partial_{\alpha} V_{\beta}$ & $a_{\alpha \beta}^{23}$ & $V_{\alpha} \star \partial_{\beta} d V \star d V$ & $a_{\alpha \beta}^{36}$ & $V \star d V \star \partial_{\alpha} d V_{\beta}$ \\
$a_{\alpha \beta}^{11}$ & $d V \star \partial_{\alpha} V_{\beta} \star d V$ & $a_{\alpha \beta}^{24}$ & $V_{\alpha} \star d V \star \partial_{\beta} d V$ & $a_{\alpha \beta}^{37}$ & $d V \star d V_{\alpha} \star d V_{\beta}$ \\
$a_{\alpha \beta}^{12}$ & $\partial_{\alpha} V_{\beta} \star d V \star d V$ & $a_{\alpha \beta}^{25}$ & $\partial_{\alpha} d V \star V \star d V_{\beta}$ & $a_{\alpha \beta}^{38}$ & $d V_{\alpha} \star d V \star d V_{\beta}$ \\
$a_{\alpha \beta}^{13}$ & $d V \star \partial_{\alpha} V \star d V_{\beta}$ & $a_{\alpha \beta}^{26}$ & $V \star \partial_{\alpha} d V \star d V_{\beta}$ & $a_{\alpha \beta}^{39}$ & $d V_{\alpha} \star d V_{\beta} \star d V$ \\
\hline
\end{tabular}

Table 1: All 4-forms with three derivatives and three gauge fields.

$$
\begin{aligned}
& +2 \partial_{\alpha} d V \star V_{\beta} \star d V+d V \star \partial_{\alpha} d V \star V_{\beta}-2 d V \star V_{\alpha} \star \partial_{\beta} d V+\partial_{\alpha} d V \star \partial_{\beta} V \star V- \\
& \left.-\partial_{\alpha} V \star \partial_{\beta} d V \star V+\partial_{\alpha} d V \star V \star \partial_{\beta} V+\partial_{\alpha} V \star V \star \partial_{\beta} d V\right] .
\end{aligned}
$$

Proceeding similarly for $s_{\star, 1} \mathcal{B}_{3}$, and taking for simplicity $x=1$, we have

$$
\begin{aligned}
s_{\star, 1} \mathcal{B}_{3}= & \frac{i}{24 \pi^{2}} \int \frac{i}{2} \omega^{\alpha \beta} \operatorname{Tr} \Lambda \star \times \\
& \times\left[V_{\alpha} \star \partial_{\beta} d V \star d V+V \star d V_{\alpha} \star \partial_{\beta} d V+\right. \\
& \quad+V \star d V \star \partial_{\alpha} d V_{\beta}+V \star \partial_{\alpha} d V \star d V_{\alpha}+d V_{\alpha} \star \partial_{\beta} d V \star V+ \\
& \left.\quad+d V \star \partial_{\alpha} d V_{\beta} \star V+\partial_{\alpha} d V \star d V \star V_{\beta}+\partial_{\alpha} d V \star d V_{\beta} \star V\right] .
\end{aligned}
$$

To simplify these expressions we introduce the notation

$$
A^{i}=-\frac{i}{24 \pi^{2}} \int \frac{i}{4} \omega^{\alpha \beta} \operatorname{Tr} \Lambda \star a_{\alpha \beta}^{i},
$$

with $a_{\alpha \beta}^{i}$ as in table 1 . Note that $\omega^{\alpha \beta} a_{\alpha \beta}^{i}$ is a 4 -form with one explicit $\omega^{\alpha \beta}$, three explicit derivatives and three noncommutative gauge fields. By "explicit" here we mean $\omega^{\prime}$ s and $\partial$ 's that are not hidden in the $\star$-product. In table 1 we have listed all such forms that can be constructed. With this notation $\stackrel{\circ}{\star, 4}-s_{\star, 1} \mathcal{B}$ reads

$$
\begin{aligned}
\stackrel{\circ}{\mathcal{A}}_{\star, 4}-s_{\star, 1} \mathcal{B}= & A^{1}-A^{2}+A^{3}+A^{4}-A^{5}+A^{6}+A^{7}-A^{8}+A^{9}-A^{10}-A^{12}- \\
& -2 A^{13}-2 A^{15}-2 A^{16}-2 A^{18}+A^{19}+A^{20}-2 A^{21}+2 A^{22}+A^{23}+ \\
& +A^{24}+2 A^{26}+2 A^{27}+2 A^{28}+2 A^{29}+2 A^{32}+2 A^{36}+2 A^{37}+2 A^{39} .
\end{aligned}
$$

Now, not all the 4-forms $w^{\alpha \beta} a_{\alpha \beta}^{i}$ in table 1 are linearly independent. To see this, consider e.g. the 5 -form $\Omega^{\beta}=\omega^{\alpha \beta} \partial_{\alpha} V \star d V \star d V$ and act on it with the inner contraction

$$
i_{\beta} \equiv i_{\partial_{\beta}}=\frac{\partial}{\partial\left(d x^{\beta}\right)} .
$$


Being a 5 -form in four dimensions, $\Omega^{\beta}$ is identically zero, and so is $i_{\beta}$ acting on it. Hence

$$
\begin{aligned}
0 & =i_{\beta}\left(\omega^{\alpha \beta} \partial_{\alpha} V \star d V \star d V\right) \\
& =\omega^{\alpha \beta}\left[\partial_{\alpha} V_{\beta} \star d V \star d V-\partial_{\alpha} V \star\left(\partial_{\beta} V-d V_{\beta}\right) \star d V-\partial_{\alpha} V \star d V \star\left(\partial_{\beta} V-d V_{\beta}\right)\right] \\
& =\omega^{\alpha \beta}\left(a_{\alpha \beta}^{12}-a_{\alpha \beta}^{3}+a_{\alpha \beta}^{15}-a_{\alpha \beta}^{2}+a_{\alpha \beta}^{14}\right),
\end{aligned}
$$

which implies the relation

$$
A^{12}-A^{3}+A^{15}-A^{2}+A^{14}=0 .
$$

This suggests that, to generate all the linear relations among the functionals $A^{i}$, it is enough to act with $i_{\beta}$ on all the 5 -forms $\Omega^{\beta}$ with one explicit $\omega^{\alpha \beta}$, three explicit derivatives and three noncommutative gauge fields. In listing the forms $\Omega^{\beta}$, two restrictions should be observed. The first one is that it is only necessary to consider 5 -forms $\Omega^{\beta}$ with at most two explicit derivatives acting on the same field, since in table 1 there is no $a_{\alpha \beta}^{i}$ with more than two explicit derivatives on the same gauge field. The second one is that whenever two explicit derivatives act on the same gauge field, they should not be both exterior derivatives. The reason for this is that 5 -forms $\Omega^{\beta}$ containing an explicit $d^{2}$ do not provide, upon acting on them with $i_{\beta}$, any relation among the $\omega^{\alpha \beta} a_{\alpha \beta}^{i}$. Indeed, since $i_{\beta} d^{2}=\partial_{\beta} d-d \partial_{\beta}$, the action of $i_{\beta}$ on a 5 -form containing an explicit $d^{2}$ yields a linear combination

$$
i_{\beta}\left(5 \text {-form with } d^{2}\right)^{\beta}=4 \text {-forms with } d^{2}+4 \text {-form with } \partial_{\beta} d-d \partial_{\beta}
$$

of 4 -forms each of which is identically zero. There are twelve different forms $\Omega^{\alpha}$ that can be constructed satisfying these restrictions on the derivatives, namely $\Omega^{\beta}=\omega^{\alpha \beta} \tilde{a}_{\alpha}$, with $\tilde{a}_{\alpha}$ given by

$$
\begin{array}{lllr}
\partial_{\alpha} V \star d V \star d V & d V_{\alpha} \star d V \star d V & \partial_{\alpha} d V \star d V \star V & \partial_{\alpha} d V \star V \star d V \\
d V \star \partial_{\alpha} V \star d V & d V \star d V_{\alpha} \star d V & d V \star \partial_{\alpha} d V \star V & V \star \partial_{\alpha} d V \star d V \\
d V \star d V \star \partial_{\alpha} V & d V \star d V \star d V_{\alpha} & d V \star V \star \partial_{\alpha} d V & V \star d V \star \partial_{\alpha} d V .
\end{array}
$$

If we act with $i_{\beta}$ on these twelve 5 -forms, we obtain the linear relations

$$
\begin{aligned}
A^{2}+A^{3}-A^{12}-A^{14}-A^{15}=0 & A^{7}+A^{19}-A^{28}-A^{31}=0 \\
A^{1}+A^{3}-A^{11}-A^{13}-A^{18}=0 & A^{8}-A^{20}-A^{29}+A^{32}=0 \\
A^{1}+A^{2}-A^{10}-A^{16}-A^{17}=0 & A^{9}+A^{21}-A^{30}-A^{33}=0 \\
A^{12}+A^{17}+A^{18}-A^{38}-A^{39}=0 & A^{4}-A^{22}-A^{25}+A^{34}=0 \\
A^{11}+A^{15}+A^{16}-A^{37}-A^{39}=0 & A^{5}+A^{23}-A^{26}-A^{35}=0 \\
A^{10}+A^{13}+A^{14}-A^{37}-A^{38}=0 & A^{6}-A^{24}-A^{27}+A^{36}=0 .
\end{aligned}
$$




\begin{tabular}{||c|c||c|c|}
\hline$b_{\alpha \beta}^{1}$ & $V_{\alpha} \star V_{\beta} \star d V \star d V$ & $b_{\alpha \beta}^{6}$ & $\partial_{\alpha} V_{\beta} \star V \star d V \star V$ \\
$b_{\alpha \beta}^{2}$ & $d V_{\alpha} \star V_{\beta} \star d V \star V$ & $b_{\alpha \beta}^{7}$ & $\partial_{\alpha} V_{\beta} \star d V \star V \star V$ \\
$b_{\alpha \beta}^{3}$ & $d V \star d V_{\alpha} \star V \star V_{\beta}$ & $b_{\alpha \beta}^{8}$ & $V_{\alpha} \star d V \star \partial_{\beta} V \star V$ \\
$b_{\alpha \beta}^{4}$ & $d V_{\alpha} \star V_{\beta} \star V \star d V$ & $b_{\alpha \beta}^{9}$ & $V_{\alpha} \star \partial_{\beta} V \star V \star d V$ \\
$b_{\alpha \beta}^{5}$ & $\partial_{\alpha} V_{\beta} \star V \star V \star d V$ & $b_{\alpha \beta}^{10}$ & $V \star d V \star \partial_{\alpha} V \star V_{\beta}$ \\
\hline
\end{tabular}

Table 2: All 4-forms with two derivatives and four gauge fields.

Solving this system of equations for $A^{i}(i=1, \ldots, 12)$ and substituting the solution in eq. (B.17), we write $\stackrel{\circ}{\mathcal{A}}_{\star, 4}-s_{\star, 1} \mathcal{B}_{3}$ in terms of the functionals $A^{i}(i=13, \ldots, 39)$, the result being

$$
\begin{aligned}
{\stackrel{\circ}{\mathcal{A}_{\star}, 4}-s_{\star, 1} \mathcal{B}_{3}=} & A^{13}+A^{14}-4 A^{15}-4 A^{16}+A^{17}+A^{18}-3 A^{21}+3 A^{22}+ \\
& +2 A^{23}+2 A^{24}+A^{25}+A^{26}+3 A^{27}+3 A^{28}+A^{29}+A^{30} \\
& +A^{31}+3 A^{32}+A^{33}-A^{34}-A^{35}+A^{36}+2 A^{37}-3 A^{38}+2 A^{39} .
\end{aligned}
$$

We have thus obtained the left-hand side of eq. (B.10) in terms of linearly independent functionals $A^{i}(i=13, \ldots, 39)$, each of which has one explicit $\omega^{\alpha \beta}$ and three explicit derivatives and has degree three in the noncommutative gauge field. It then follows that, for eq. (B.10) to have a solution, $\mathcal{B}_{4}$ on the right-hand side must be a linear combination of functionals

$$
B^{r}=-\frac{i}{24 \pi^{2}} \int \frac{i}{4} \omega^{\alpha \beta} \operatorname{Tr} b_{\alpha \beta}^{r},
$$

with $b_{\alpha \beta}^{r}$ a 4-form of order two in explicit derivatives and four in the noncommutative gauge field. With some patience, it can be seen that there are forty such functionals $B^{r}$ whose $s_{\star, 0}$ variation is not zero. Thirty of them can be written as linear combinations of the functionals $B^{r}$ whose $b_{\alpha \beta}^{r}$ are collected in table 2. To illustrate that this is indeed so, let us consider as an example

$$
B=-\frac{i}{24 \pi^{2}} \int \frac{i}{4} \omega^{\alpha \beta} \operatorname{Tr} b_{\alpha \beta} \quad b_{\alpha \beta}=V \star V_{\alpha} \star \partial_{\beta} V \star d V .
$$

Clearly, this $b_{\alpha \beta}$ in not in table 2. However, using that

(a) both $\operatorname{Tr}$ and the integral of a Moyal product of functions are cyclic,

(b) that $\partial_{\alpha}=\left\{i_{\alpha}, d\right\}$, and

(c) that $i_{\beta}(d V) \star d V \star V \star V_{\alpha}=-d V \star i_{\beta}\left(d V \star V \star V_{\alpha}\right)$,

and integrating by parts and neglecting total derivatives, we have

$$
\begin{aligned}
B & \stackrel{(\mathrm{a}, \mathrm{b})}{=} \frac{i}{24 \pi^{2}} \int \frac{i}{4} \omega^{\alpha \beta} \operatorname{Tr}\left(i_{\beta} d+d i_{\beta}\right) V \star d V \star V \star V_{\alpha} \\
& \stackrel{(\mathrm{c}, \mathrm{d})}{=}-\frac{i}{24 \pi^{2}} \int \frac{i}{4} \omega^{\alpha \beta} \operatorname{Tr}\left[d V \star i_{\beta}\left(d V \star V \star V_{\alpha}\right)+i_{\beta} V \star d\left(d V \star V \star V_{\alpha}\right)\right] \\
& \stackrel{(\mathrm{a})}{=}-\frac{i}{24 \pi^{2}} \int \frac{i}{4} \omega^{\alpha \beta} \operatorname{Tr}\left(b_{\alpha \beta}^{8}+b_{\alpha \beta}^{3}+b_{\alpha \beta}^{2}\right) \\
& =B^{8}+B^{3}+B^{2} .
\end{aligned}
$$


Similarly, any other functional $B$ whose $b_{\alpha \beta}$ is not in table 2 can be expressed as a linear combination of functionals $B^{r}$ with $b_{\alpha \beta}^{r}$ in table 2. It then follows that it is enough to write for $\mathcal{B}_{4}$

$$
\mathcal{B}_{4}=\sum_{r=1}^{10} c_{r} B^{r} .
$$

To solve eq. (B.10) we need the $s_{\star, 0}$ variation of $\mathcal{B}_{4}$. Acting with $s_{\star, 0}$ on $(\mathrm{B} .20)$ and writing the result in terms of the linearly independent functionals $A^{i}$, corresponding to $i=13, \ldots, 39$, we obtain

$$
\begin{aligned}
s_{\star, 0} \mathcal{B}_{4}= & \left(-c_{1}-c_{2}+c_{5}+c_{6}+2 c_{9}\right) A^{13}+ \\
& +\left(-c_{1}-c_{4}+c_{5}+c_{6}+c_{8}+3 c_{9}\right) A^{14} \\
& +\left(-2 c_{3}-c_{5}+c_{7}+c_{8}+c_{10}\right)\left(A^{15}+A^{16}\right)+ \\
& +\left(c_{1}-2 c_{2}-c_{4}-c_{6}-c_{7}+2 c_{8}-c_{9}+c_{10}\right) A^{17}+ \\
& +\left(c_{1}-c_{2}-2 c_{4}-c_{6}-c_{7}+c_{8}+c_{10}\right) A^{18}+ \\
& +\left(c_{1}+c_{8}-c_{9}\right)\left(A^{19}+A^{20}\right)+ \\
& +\left(c_{3}-c_{8}\right)\left(-A^{21}+A^{22}+A^{27}+A^{28}\right)+ \\
& +\left(c_{1}-c_{2}-c_{4}\right) A^{23}+ \\
& +\left(c_{1}-c_{2}-c_{4}+c_{8}+c_{10}\right) A^{24}- \\
& -c_{2}\left(A^{25}+A^{29}\right)+ \\
& +\left(-c_{4}+c_{9}+c_{10}\right)\left(A^{26}+A^{30}\right)+ \\
& +\left(c_{2}+c_{7}-c_{8}\right) A^{31}+ \\
& +\left(c_{3}+c_{5}\right) A^{32}+ \\
& +\left(c_{2}-c_{6}-c_{9}\right) A^{33}+ \\
& +\left(c_{4}+c_{6}+c_{10}\right) A^{34}+ \\
& +\left(c_{3}-c_{7}-c_{8}-c_{10}\right) A^{35}+ \\
& +\left(c_{4}-c_{5}-c_{9}\right) A^{36}+ \\
& +\left(c_{1}+c_{3}-c_{4}-c_{6}-c_{7}-c_{9}\right) A^{37}+ \\
& +\left(c_{2}-c_{3}+c_{4}-c_{5}+c_{7}-c_{9}\right) A^{38}+ \\
& +\left(-c_{1}+c_{3} 4+c_{4}+c_{5}+c_{6}+c_{8}-c_{9}+c_{10}\right) A^{39} . \\
& +
\end{aligned}
$$

Substituting now eqs. (B.18) and (B.21) in eq. (B.10) and equating the coefficients of $A^{i}(i=13, \ldots, 39)$ on both sides, we obtain a system of 21 equations with unknowns $c_{1}, \ldots, c_{10}$. Its solution is

$$
\begin{array}{llllr}
c_{1}=y+z & c_{2}=-1 & c_{3}=3-z & c_{4}=-1+y+z & c_{5}=z \\
c_{6}=-y & c_{7}=2-z & c_{8}=-z & c_{9}=y & c_{10}=z,
\end{array}
$$

where $y$ and $z$ are arbitrary parameters. This provides a two-parameter family of functionals $\mathcal{B}_{4}$ for which eq. (B.10) holds. Note that if we take $y=z=0$, then $\mathcal{B}_{4}$ only has four terms. 


\section{B.2 Calculation of $\mathcal{B}_{5}$ and $\mathcal{B}_{6}$}

One may proceed analogously as for $\mathcal{B}_{4}$ and explicitly compute $\mathcal{B}_{5}$ and $\mathcal{B}_{6}$. Here, instead, we present an alternative method which uses cohomological techniques. To apply them we shall employ the approach of ref. [23 which introduces gauge fields $v_{\mu}^{A}$ and ghost fields $\lambda^{A}$ not only for the Lie algebra $\mathfrak{g}$ of the gauge group $G$ but also for the whole enveloping algebra $\mathcal{U}=\left\{T_{A}\right\}=\left\{T_{\bar{a}}, T_{i}\right\}$ in which $V_{\mu}$ and $\Lambda$ take values. Here the index $\bar{a}$ runs over the elements of $\mathfrak{g}$, so that in the notation of section 2 one has $\left\{T_{\bar{a}}\right\}=\left\{\left(T^{k}\right)^{a}, T^{l}\right\}$, while the index $i$ runs over the complementary elements of $\mathcal{U}$. As shown in ref. [23, the standard Seiberg-Witten map can be extended to include $\mathcal{U}$-valued fields $v_{\mu}$ and $\lambda$ satisfying

$$
\begin{aligned}
s v_{\mu}^{A} & =\partial_{\mu} \lambda^{A}+f_{B C}{ }^{A} v_{\mu}^{B} \lambda^{C} \\
s \lambda^{A} & =\frac{1}{2} \lambda^{B} \lambda^{C} f_{C B}{ }^{A}
\end{aligned}
$$

with $f_{A B}{ }^{C}$ the structure constants of the Lie algebra $\mathcal{U}$, given by $\left[T_{A}, T_{B}\right]=f_{A B}{ }^{C} T_{C}$. Of course, $\mathfrak{g}$ being a subalgebra of $\mathcal{U}$ means $f_{\bar{a} \bar{b}}{ }^{i}=0$ and implies that the BRS transformations above are subject to the truncation conditions

$$
\begin{gathered}
\left.s v_{\mu}^{A}\right|_{v_{\mu}^{i}=\lambda^{i}=0}= \begin{cases}\partial_{\mu} \lambda^{\bar{a}}+f_{\bar{b} \bar{c}}^{\bar{a}} v_{\mu}^{\bar{b}} \lambda^{\bar{c}} & \text { if } A=\bar{a} \\
f_{\bar{b} \bar{c}}{ }^{i} v_{\mu}^{\bar{b}} \lambda^{\bar{c}}=0 & \text { if } A=i\end{cases} \\
\left.s \lambda^{A}\right|_{v_{\mu}^{i}=\lambda^{i}=0}= \begin{cases}\frac{1}{2} \lambda^{\bar{b}} \lambda^{\bar{c}} f_{\bar{b} \bar{c}}^{\bar{a}} & \text { if } A=\bar{a} \\
\frac{1}{2} \lambda^{\bar{b}} \lambda^{\bar{c}} f_{\bar{c} \bar{b}}^{i}=0 & \text { if } A=i .\end{cases}
\end{gathered}
$$

The extended Seiberg-Witten map is defined by demanding

$$
s_{\star} V_{\mu}^{A}=s V_{\mu}^{A} \quad s_{\star} \Lambda^{A}=s \Lambda^{A}
$$

subject to the usual boundary conditions and with $s_{\star}$ defined by

$$
s_{\star} V_{\mu}^{A}=\partial_{\mu} \Lambda^{A}+f_{B C}{ }^{A} V_{\mu}^{B} \Lambda^{C} \quad s_{\star} \Lambda^{A}=\frac{1}{2} \Lambda^{B} \Lambda^{C} f_{C B}{ }^{A} .
$$

By setting in it all fields $v_{\mu}^{i}$ and $\lambda^{i}$ to zero, the standard Seiberg-Witten map is recovered. Furthermore, the truncation conditions imply that all formulas that hold for $\mathcal{U}$-valued fields $v_{\mu}^{A}$ and $\lambda^{A}$ will also hold for $\mathfrak{g}$-valued fields $v_{\mu}^{\bar{a}}$ and $\lambda^{\bar{a}}$, and in particular eq. (B.2) that we want to prove. The idea is then to demonstrate eq. (B.2) for the extended SeibergWitten map.

We start from the fact that $\mathcal{A}_{\star}$ satisfies the anomaly consistency condition $s_{\star} \mathcal{A}_{\star}=0$ which follows from eq. (B.2) because of $s_{\star}^{2}=0$. In terms of the commutative fields $v_{\mu}^{A}$ and $\lambda^{A}$, one has $s \mathcal{A}_{\star}=0$. This implies $s \stackrel{\circ}{\star}_{\star}=0$ since $s$ commutes with the logarithmic 
derivative with respect to $t$. Using (B.26) again, one concludes $s_{\star} \stackrel{\circ}{\star}_{\star}=0$ which decomposes into

$$
\begin{aligned}
& s_{\star, 0} \stackrel{\circ}{\mathcal{A}}_{\star, 3}=0 \\
& s_{\star, 0} \stackrel{\circ}{\mathcal{A}}_{\star, 4}+s_{\star, 1} \stackrel{\circ}{\mathcal{A}}_{\star, 3}=0 \\
& s_{\star, 0}{\stackrel{\circ}{\mathcal{A}_{\star}, 5}}+s_{\star, 1} \stackrel{\circ}{\mathcal{A}}_{\star, 4}=0 \\
& s_{\star, 0} \stackrel{\circ}{\mathcal{A}}_{\star, 6}+s_{\star, 1} \stackrel{\circ}{\mathcal{A}}_{\star, 5}=0 \\
& s_{\star, 1} \stackrel{\circ}{\mathcal{A}}_{\star, 6}=0 .
\end{aligned}
$$

In the previous subsection we have shown by explicit computation that (B.28) and (B.29) imply $\stackrel{\circ}{\star}_{\star, 3}=s_{\star, 0} \mathcal{B}_{3}$ and $\stackrel{\circ}{\mathcal{A}}_{\star, 4}=s_{\star, 0} \mathcal{B}_{4}+s_{\star, 1} \mathcal{B}_{3}$. We shall now show by cohomological means that the remaining equations imply $\stackrel{\circ}{\mathcal{A}}_{\star, 5}=s_{\star, 0} \mathcal{B}_{5}+s_{\star, 1} \mathcal{B}_{4}, \stackrel{\circ}{\mathcal{A}}_{\star, 6}=s_{\star, 0} \mathcal{B}_{6}+s_{\star, 1} \mathcal{B}_{5}$ and $s_{\star, 1} \mathcal{B}_{6}=0$, which will complete the proof of equations (B.9) to (B.13).

To that end we first derive a result on the cohomology of $s_{\star, 0}$ in the space $\mathcal{F}_{\star}$ of integrated $\star$-polynomials in the fields $V_{\mu}^{A}, \Lambda^{A}$ and their derivatives. An element of this space is a linear combination, with coefficients that may depend on $\omega^{\alpha \beta}$, of terms of the form

$$
\int d^{4} x a_{1} \star a_{2} \star \ldots \star a_{n},
$$

with $n$ finite and each $a_{i}$ one of our basic variables $\left(V_{\mu}^{A}, \Lambda^{A}\right.$ and their derivatives),

$$
a_{i} \in\left\{V_{\mu}^{A}, \Lambda^{A}, \partial_{\mu} V_{\nu}^{A}, \partial_{\mu} \Lambda^{A}, \partial_{\mu} \partial_{\nu} V_{\rho}^{A}, \partial_{\mu} \partial_{\nu} \Lambda^{A}, \ldots\right\}
$$

It is obvious why this cohomology is relevant to the present case. Using the result ${\stackrel{\circ}{\mathcal{A}_{\star} 4}}=$ $s_{\star, 0} \mathcal{B}_{4}+s_{\star, 1} \mathcal{B}_{3}$ from Subappendix B.1 in eq. (B.30) and noting that $s_{\star, 1}^{2}=0$, we obtain $s_{\star, 0}\left(\stackrel{\circ}{\mathcal{A}}_{\star, 5}-s_{\star, 1} \mathcal{B}_{4}\right)=0$, with $\stackrel{\circ}{\mathcal{A}}_{\star, 5}-s_{\star, 1} \mathcal{B}_{4}$ obviously in $\mathcal{F}_{\star}$. Our aim is to show that this implies $\stackrel{\circ}{\star}_{\star, 5}-s_{\star, 1} \mathcal{B}_{4}=s_{\star, 0} \mathcal{B}_{5}$ for some $\mathcal{B}_{5} \in \mathcal{F}_{\star}$, or in other words that $\stackrel{\circ}{\star}_{\star, 5}-s_{\star, 1} \mathcal{B}_{4}$ is trivial in the $s_{\star, \sigma}$ cohomology in $\mathcal{F}_{\star}$. Assume that we have shown this. Inserting the result in (B.31) and proceeding similarly yields $s_{\star, 0}\left(\stackrel{\circ}{\star}_{\star, 6}-s_{\star, 1} \mathcal{B}_{5}\right)=0$. Again, we want to show that $\stackrel{\circ}{\mathcal{A}}_{\star, 6}-s_{\star, 1} \mathcal{B}_{5}=s_{\star, 0} \mathcal{B}_{6}$ for some $\mathcal{B}_{6} \in \mathcal{F}_{\star}$ and thus that $\stackrel{\circ}{\mathcal{A}}_{\star, 6}-s_{\star, 1} \mathcal{B}_{5}$ is also trivial in the $s_{\star, \sigma}$ cohomology in $\mathcal{F}_{\star}$. Note that $(\overline{B .9})$ and $(\overline{B .10})$ actually express analogous results, namely the triviality of $\stackrel{\circ}{\star}_{\star, 3}$ and $\stackrel{\circ}{\star}_{\star, 4}-s_{\star, 1} \mathcal{B}_{3}$ in the same cohomology. However, as it will become clear below, they cannot be proved by means of the result on the cohomology for $s_{\star, 0}$ in $\mathcal{F}_{\star}$ that we derive in the sequel and therefore have to be shown by other methods.

To examine the $s_{\star, \sigma}$ cohomology in $\mathcal{F}_{\star}$ we adapt methods developed in ref. [28 for the computation of the cohomology of $s_{0}$. We first derive a result on the $s_{\star, \sigma}$ cohomology in the space $\mathcal{P}_{\star}$ of non-integrated $\star$-polynomials. For that purpose we introduce the following variables $u^{\ell}, v^{\ell}$ and $w^{i}$ :

$$
\left\{u^{\ell}\right\}=\left\{V_{\mu}^{A}, \partial_{(\mu} V_{\nu)}^{A}, \ldots, \partial_{\left(\mu_{1}\right.} \ldots \partial_{\mu_{k}} V_{\left.\mu_{k+1}\right)}^{A}, \ldots\right\}
$$




$$
\begin{aligned}
\left\{v^{\ell}\right\} & =\left\{s_{\star, 0} u^{\ell}\right\}=\left\{\partial_{\mu} \Lambda^{A}, \partial_{(\mu} \partial_{\nu)} \Lambda^{A}, \ldots, \partial_{\left(\mu_{1}\right.} \ldots \partial_{\left.\mu_{k+1}\right)} \Lambda^{A}, \ldots\right\} \\
\left\{w^{i}\right\} & =\left\{\Lambda^{A}, \partial_{[\mu} V_{\nu]}^{A}, \ldots, \partial_{\mu_{1}} \ldots \partial_{\mu_{k}} \partial_{[\mu} V_{\nu]}^{A}, \ldots\right\} .
\end{aligned}
$$

Evidently every $\star$-polynomial in the fields $V_{\mu}^{A}, \Lambda^{A}$ and their derivatives can be expressed as a $\star$-polynomial in the variables $u^{\ell}, v^{\ell}, w^{i}$ and vice versa. ${ }^{1}$ On non-integrated $\star$-monomials in $u^{\ell}, v^{\ell}, w^{i}$ we define the operation $\varrho$ through

$$
\begin{aligned}
\varrho\left(\hat{a}_{1} \star \hat{a}_{2} \star \ldots \star \hat{a}_{n}\right)= & \frac{1}{n}\left(u^{\ell} \frac{\partial \hat{a}_{1}}{\partial v^{\ell}}\right) \star \hat{a}_{2} \star \ldots \star \hat{a}_{n}+ \\
& +\frac{1}{n} \sum_{i=2}^{n-1}(-)^{\left|\hat{a}_{1}\right|+\left|\hat{a}_{2}\right|+\cdots+\left|\hat{a}_{i-1}\right|} \hat{a}_{1} \star \ldots \star \hat{a}_{i-1} \star\left(u^{\ell} \frac{\partial \hat{a}_{i}}{\partial v^{\ell}}\right) \star \hat{a}_{i+1} \star \ldots \star \hat{a}_{n}+ \\
& +\frac{1}{n}(-)^{\left|\hat{a}_{1}\right|+\left|\hat{a}_{2}\right|+\cdots+\left|\hat{a}_{n-1}\right|} \hat{a}_{1} \star \hat{a}_{2} \star \ldots \star \hat{a}_{n-1} \star\left(u^{\ell} \frac{\partial \hat{a}_{n}}{\partial v^{\ell}}\right),
\end{aligned}
$$

where $\hat{a}_{i}$ is any of the variables $u^{\ell}, v^{\ell}, w^{i}$,

$$
\hat{a}_{i} \in\left\{u^{\ell}, v^{\ell}, w^{i}\right\},
$$

and $\left|\hat{a}_{i}\right|$ is the Grassmann parity of $\hat{a}_{i}$, which is 0 for $V_{\mu}^{A}$ and its derivatives, and 1 for the $\Lambda^{A}$ and its derivatives. Extending the definition of $\varrho$ by linearity from $\star$-monomials to $\star$-polynomials, we have that the anticommutator of $s_{\star, 0}$ and $\varrho$ evaluated on an arbitrary $\star$-polynomial $p_{\star}(u, v, w) \in \mathcal{P}_{\star}$ gives the difference

$$
\left\{s_{\star}, 0, \varrho\right\} p_{\star}(u, v, w)=p_{\star}(u, v, w)-p_{\star}(0,0, w),
$$

where $p_{\star}(0,0, w)$ denotes the $\star$-polynomial that arises from $p_{\star}(u, v, w)$ by setting to zero all $u^{\ell}$ and $v^{\ell}$ before evaluating the star-products - for example, for $p_{\star}=V_{\mu}^{A} \star V_{\nu}^{B}$ one has $p_{\star}(0,0, w)=0$. Applying now eq. $(\overline{\mathrm{B} .36})$ to an $s_{\star, 0}$-closed $\star$-polynomial, i.e. to a $p_{\star}$ satisfying $s_{\star, 0} p_{\star}=0$, and using that all $w^{i}$ are $s_{\star, 0}$-closed, we obtain

$$
s_{\star, 0} p_{\star}(u, v, w)=0 \quad \Leftrightarrow \quad p_{\star}(u, v, w)=p_{\star}(0,0, w)+s_{\star, 0} \varrho p_{\star}(u, v, w) .
$$

In particular, an $s_{\star, \sigma}$ closed $\star$-polynomial $p_{\star}(u, v, w)$ with $p_{\star}(0,0, w)=0$ is the $s_{\star, \sigma}$-variation of the star-polynomial $\varrho p_{\star}(u, v, w)$.

Result (B.37) cannot be used directly for our purposes since it applies only to $\star$ polynomials but not to integrated $\star$-polynomials, which is what we had initially. This makes a difference because, by definition, an integrated $\star$-polynomial is $s_{\star, \sigma}$ closed when the $s_{\star, \sigma}$ transformation of its integrand is a total divergence:

$$
s_{\star, 0} f_{\star}=0 \quad \text { with } \quad f_{\star}=\int d^{4} x p_{\star} \quad \Leftrightarrow \quad s_{\star, 0} p_{\star}=\partial_{\mu} \omega^{\mu} \quad \text { for some } \quad \omega^{\mu} .
$$

Since $\varrho$ does not commute with $\partial_{\mu}$ we cannot directly apply the result above to this case. To escape this problem we consider the variational derivatives of the equation $s_{\star, 0} f_{\star}=0$ with respect to $V_{\mu}^{A}$ and $\Lambda^{A}$. This yields

$$
s_{\star, 0} f_{\star}=0, \quad f_{\star} \in \mathcal{F}_{\star} \quad \Rightarrow \quad s_{\star, 0} \frac{\delta f_{\star}}{\delta V_{\mu}^{A}}=0, \quad s_{\star, 0} \frac{\delta f_{\star}}{\delta \Lambda^{A}}+\partial_{\mu} \frac{\delta f_{\star}}{\delta V_{\mu}^{A}}=0 .
$$

\footnotetext{
${ }^{1}$ The set of $w$ 's is actually overcomplete because the $w$ 's are not all linearly independent owing to the identities $\partial_{[\mu} \partial_{\nu} V_{\rho]}=0$ and their derivatives. However this does not matter to our arguments.
} 
It can be readily checked that the variational derivative of any element $f_{\star} \in \mathcal{F}_{\star}$ with respect to $V_{\mu}^{A}$ or $\Lambda^{A}$ is a $\star$-polynomial in $\mathcal{P}_{\star}$. Suppose now that $\delta f_{\star} / \delta V_{\mu}^{A}$ vanishes at $u^{\ell}=v^{\ell}=0$ in the sense explained above. Using the first equation in (B.38) and eq. (B.37) we then conclude that $\delta f_{\star} / \delta V_{\mu}^{A}$ is the $s_{\star, 0}$ variation of $\varrho\left(\delta f_{\star} / \delta V_{\mu}^{A}\right)$ :

$$
\left[\frac{\delta f_{\star}}{\delta V_{\mu}^{A}}\right](0,0, w)=0 \quad \Rightarrow \quad \frac{\delta f_{\star}}{\delta V_{\mu}^{A}}=s_{\star, 0} \varrho \frac{\delta f_{\star}}{\delta V_{\mu}^{A}} .
$$

Using this in the second equation in $(\mathrm{B.38})$ we obtain

$$
s_{\star, 0}\left(\frac{\delta f_{\star}}{\delta \Lambda^{A}}+\partial_{\mu} \varrho \frac{\delta f_{\star}}{\delta V_{\mu}^{A}}\right)=0 .
$$

Applying (B.37) once again we conclude that the term in parentheses is $s_{\star, 0} \varrho(\ldots)$ provided it vanishes at $u^{\ell}=v^{\ell}=0$ in the sense above. Note that here $\varrho(\ldots)$ has ghost number $\operatorname{gh}\left(f_{\star}\right)-2$, with $\operatorname{gh}\left(f_{\star}\right)$ the ghost number of $f_{\star}$ and $\operatorname{gh}(V)=0$ and $\operatorname{gh}(\Lambda)=1$. Since $\star$-polynomials $p_{\star}(u, v, w)$ have non-negative ghost numbers, $\varrho(\ldots)$ vanishes when $f_{\star}$ has ghost number 1 , which is the case we are interested in. We thus conclude that

$$
\left[\frac{\delta f_{\star}}{\delta \Lambda^{A}}+\partial_{\mu} \varrho \frac{\delta f_{\star}}{\delta V_{\mu}^{A}}\right](0,0, w)=0, \quad \operatorname{gh}\left(f_{\star}\right)=1 \quad \Rightarrow \quad \frac{\delta f_{\star}}{\delta \Lambda^{A}}=-\partial_{\mu} \varrho \frac{\delta f_{\star}}{\delta V_{\mu}^{A}} .
$$

Finally we reconstruct $f_{\star}$ from its variational derivatives, neglecting integrated divergences, using the general formula

$$
f_{\star}[V, \Lambda]=\int d^{4} x \int_{0}^{1} \frac{d \tau}{\tau}\left(V_{\mu}^{A} \star \frac{\delta f_{\star}}{\delta V_{\mu}^{A}}+\Lambda^{A} \star \frac{\delta f_{\star}}{\delta \Lambda^{A}}\right)[\tau V, \tau \Lambda],
$$

valid for every functional $f_{\star}$. Using eqs. $(\mathrm{B.39})$ and $(\mathrm{B.40})$ in $(\mathrm{B} .41$ we obtain

$$
\begin{aligned}
f_{\star}[V, \Lambda] & =\int d^{4} x \int_{0}^{1} \frac{d \tau}{\tau}\left(V_{\mu}^{A} \star s_{\star, 0} \varrho \frac{\delta f_{\star}}{\delta V_{\mu}^{A}}-\Lambda^{A} \star \partial_{\mu} \varrho \frac{\delta f_{\star}}{\delta V_{\mu}^{A}}\right)[\tau V, \tau \Lambda] \\
& =\int d^{4} x \int_{0}^{1} \frac{d \tau}{\tau}\left(V_{\mu}^{A} \star s_{\star, 0} \varrho \frac{\delta f_{\star}}{\delta V_{\mu}^{A}}+\left(s_{\star, 0} V_{\mu}^{A}\right) \star \varrho \frac{\delta f_{\star}}{\delta V_{\mu}^{A}}\right)[\tau V, \tau \Lambda] \\
& =s_{\star, 0} \int d^{4} x \int_{0}^{1} \frac{d \tau}{\tau}\left(V_{\mu}^{A} \star \varrho \frac{\delta f_{\star}}{\delta V_{\mu}^{A}}\right)[\tau V, \tau \Lambda]
\end{aligned}
$$

where we have used integration by parts and $s_{\star, 0} V_{\mu}^{A}=\partial_{\mu} \Lambda^{A}$. We have thus shown that

$$
\begin{gathered}
s_{\star, 0} f_{\star}=0, \quad\left[\frac{\delta f_{\star}}{\delta V_{\mu}^{A}}\right](0,0, w)=\left[\frac{\delta f_{\star}}{\delta \Lambda^{A}}+\partial_{\mu} \varrho \frac{\delta f_{\star}}{\delta V_{\mu}^{A}}\right](0,0, w)=0 \\
\Rightarrow \quad f_{\star}=s_{\star, 0} \int d^{4} x \int_{0}^{1} \frac{d \tau}{\tau}\left(V_{\mu}^{A} \star \varrho \frac{\delta f_{\star}}{\delta V_{\mu}^{A}}\right)[\tau V, \tau \Lambda],
\end{gathered}
$$

which is the result for the $s_{\star, \sigma}$ cohomology in $\mathcal{F}_{\star}$ we will use to prove eqs. (B.30)-(B.32). 
Consider now $\stackrel{\circ}{\mathcal{A}}_{\star, 5}-s_{\star, 1} \mathcal{B}_{4}$. It is an $s_{\star, 0}$ closed integrated $\star$-polynomial with ghost number 1 whose integrand is order 5 in the fields $V_{\mu}^{A}$ and $\Lambda^{A}$, has mass dimension 4 - recall that $\operatorname{dim}\left(V_{\mu}\right)=\operatorname{dim}\left(\partial_{\mu}\right)=1, \operatorname{dim}(\Lambda)=0, \operatorname{dim}\left(\omega^{\alpha \beta}\right)=-2$ - and contains one explicit $\omega^{\alpha \beta}$. It follows that the integrand is a linear combination of $\star$-monomials $\omega^{\alpha \beta} a_{1} \star \ldots \star a_{5}$, where it can be assumed that one of the $a_{i}$ is an undifferentiated $\Lambda$ (for one can remove all derivatives from $\Lambda$ using integrations by parts, if necessary) while the remaining $a_{i}{ }^{\prime}$ s are either of type $\{V, V, \partial V, \partial V\}$ or $\{V, V, V, \partial \partial V\}$. It is easy to verify that this in turn implies

$$
\begin{array}{r}
{\left[\frac{\delta\left(\stackrel{\circ}{\star}_{\star, 5}-s_{\star, 1} \mathcal{B}_{4}\right)}{\delta V_{\mu}^{A}}\right](0,0, w)=0} \\
{\left[\frac{\delta\left(\stackrel{\circ}{\star}_{\star, 5}-s_{\star, 1} \mathcal{B}_{4}\right)}{\delta \Lambda^{A}}+\partial_{\mu} \varrho \frac{\delta\left(\stackrel{\circ}{\star}_{\star, 5}-s_{\star, 1} \mathcal{B}_{4}\right)}{\delta V_{\mu}^{A}}\right](0,0, w)=0 .}
\end{array}
$$

Eq. (B.42) can then be used and yields $\stackrel{\circ}{\mathcal{A}}_{\star, 5}-s_{\star, 1} \mathcal{B}_{4}=s_{\star, 0} \mathcal{B}_{5}$, with

$$
\mathcal{B}_{5}=\int d^{4} x \int_{0}^{1} \frac{d \tau}{\tau}\left(V_{\mu}^{A} \star \varrho \frac{\delta\left(\stackrel{\circ}{\star}_{\star, 5}-s_{\star, 1} \mathcal{B}_{4}\right)}{\delta V_{\mu}^{A}}\right)[\tau V, \tau \Lambda] .
$$

This proves eq. (B.11) for $\mathcal{U}$-valued fields, hence for $\mathfrak{g}$-valued fields, as we wanted to show.

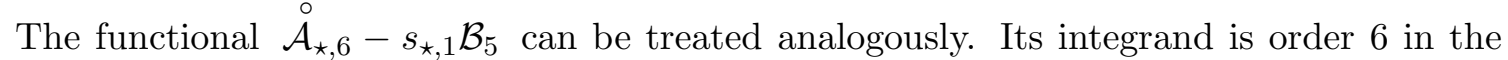
fields, has mass dimension 4 , ghost number 1 and one explicit $\omega^{\alpha \beta}$. It is thus a linear combination of $\star$-monomials $\omega^{\alpha \beta} a_{1} \star \ldots \star a_{6}$, where it can be assumed that the set of $a_{i}$ has the structure $\{\Lambda, V, V, V, V, \partial V\}$. This makes it obvious that $\stackrel{\circ}{\star}_{\star, 6}-s_{\star, 1} \mathcal{B}_{5}$ satisfies

$$
\begin{array}{r}
{\left[\frac{\delta\left(\stackrel{\circ}{\star}_{\star, 6}-s_{\star, 1} \mathcal{B}_{5}\right)}{\delta V_{\mu}^{A}}\right](0,0, w)=0} \\
{\left[\frac{\delta\left({\left.\stackrel{\circ}{\mathcal{A}_{\star}, 6}-s_{\star, 1} \mathcal{B}_{5}\right)}_{\delta \Lambda^{A}}+\partial_{\mu} \varrho \frac{\delta\left(\stackrel{\circ}{\star}_{\star, 6}-s_{\star, 1} \mathcal{B}_{5}\right)}{\delta V_{\mu}^{A}}\right](0,0, w)}{}=0 .\right.}
\end{array}
$$

Eq. (B.42) then implies $\stackrel{\circ}{\star}_{\star, 6}-s_{\star, 1} \mathcal{B}_{5}=s_{\star, 0} \mathcal{B}_{6}$, with

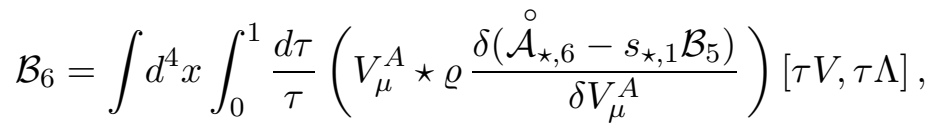

which proves eq. (B.12). Finally we have to show that eq. (B.13) holds. This is very easy. The integrand of $\mathcal{B}_{6}$ is a $\star$-polynomial of order 6 in the fields, has mass dimension 4 , ghost number 0 and one explicit $\omega^{\alpha \beta}$. It is thus a linear combination of $\star$-monomials $\omega^{\alpha \beta} a_{1} \star \ldots \star a_{6}$, where all $a_{i}$ are undifferentiated $V^{\prime}$ s. Furthermore, by construction, it can be written as a trace $\operatorname{Tr}$. The latter implies already $s_{\star, 1} \mathcal{B}_{6}=0$, since

$$
s_{\star, 1} \operatorname{Tr}\left(V_{\mu_{1}} \star \ldots \star V_{\mu_{6}}\right)=\operatorname{Tr}\left[V_{\mu_{1}} \star \ldots \star V_{\mu_{6}}, \Lambda\right]_{\star}
$$

is a divergence. 
We close by remarking that eq. (B.42) cannot be used to prove that $\stackrel{\circ}{\star, 3}_{\text {(B. }}$ and $\stackrel{\circ}{\star \star A, 4}^{-}$ $s_{\star, 1} \mathcal{B}_{3}$ are trivial in the $s_{\star, \sigma}$ cohomology in $\mathcal{F}_{\star}$ because the $\frac{\delta}{\delta V_{\mu}^{A}}$ and $\frac{\delta}{\delta \Lambda^{A}}+\partial_{\mu} \varrho \frac{\delta}{\delta V_{\mu}^{A}}$ acting on them do not vanish at $u^{\ell}=v^{\ell}=0$ in the sense explained above, contrary to what

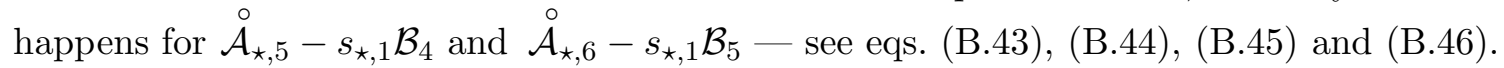

\section{References}

[1] J. Madore, S. Schraml, P. Schupp and J. Wess, Gauge theory on noncommutative spaces, Eur. Phys. J. C 16 (2000) 161 hep-th/0001203.

[2] B. Jurco, S. Schraml, P. Schupp and J. Wess, Enveloping algebra valued gauge transformations for non-abelian gauge groups on non-commutative spaces, Eur. Phys. J. C 17 (2000) 521 hep-th/0006246.

[3] N. Seiberg and E. Witten, String theory and noncommutative geometry, J. High Energy Phys. 09 (1999) 032 hep-th/9908142.

[4] B. Jurco, P. Schupp and J. Wess, Nonabelian noncommutative gauge theory via noncommutative extra dimensions, Nucl. Phys. B 604 (2001) 148 hep-th/0102129.

[5] B. Jurco, L. Moller, S. Schraml, P. Schupp and J. Wess, Construction of non-abelian gauge theories on noncommutative spaces, Eur. Phys. J. C 21 (2001) 383 hep-th/0104153.

[6] X. Calmet, B. Jurco, P. Schupp, J. Wess and M. Wohlgenannt, The standard model on non-commutative space-time, Eur. Phys. J. C 23 (2002) 363 hep-ph/0111115.

[7] S.M. Carroll, J.A. Harvey, V.A. Kostelecky, C.D. Lane and T. Okamoto, Noncommutative field theory and Lorentz violation, Phys. Rev. Lett. 87 (2001) 141601 hep-th/0105082.

[8] C.E. Carlson, C.D. Carone and R.F. Lebed, Bounding noncommutative QCD, Phys. Lett. B $\mathbf{5 1 8}(2001) 201$ hep-ph/0107291.

[9] W. Behr et al., The $z \rightarrow \gamma \gamma$, gg decays in the noncommutative standard model, hep-ph/0202121.

[10] C.E. Carlson, C.D. Carone and R.F. Lebed, Supersymmetric noncommutative QED and Lorentz violation, Phys. Lett. B 549 (2002) 337 hep-ph/0209077.

[11] P. Schupp, J. Trampetic, J. Wess and G. Raffelt, The photon neutrino interaction in non-commutative gauge field theory and astrophysical bounds, hep-ph/0212292.

[12] P. Minkowski, P. Schupp and J. Trampetic, Non-commutative '*-charge radius' and '*-dipole moment' of the neutrino, hep-th/0302175.

[13] P. Aschieri, B. Jurco, P. Schupp and J. Wess, Non-commutative GUTs, standard model and C, P, T, Nucl. Phys. B 651 (2003) 45 hep-th/0205214.

[14] G. Barnich and M. Henneaux, Renormalization of gauge invariant operators and anomalies in Yang-Mills theory, Phys. Rev. Lett. 72 (1994) 1588 hep-th/9312206.

[15] G. Barnich, F. Brandt and M. Henneaux, Local BRST cohomology in the antifield formalism, 2. Application to Yang-Mills theory, Commun. Math. Phys. 174 (1995) 93 hep-th/9405194.

[16] G. Barnich, F. Brandt and M. Henneaux, Local BRST cohomology in gauge theories, Phys. Rept. 338 (2000) 439 hep-th/0002245. 
[17] R. Wulkenhaar, Non-renormalizability of theta-expanded noncommutative QED, J. High Energy Phys. 03 (2002) 024 hep-th/0112248.

[18] C.P. Martin, The gauge anomaly and the Seiberg-Witten map, Nucl. Phys. B 652 (2003) 72 hep-th/0211164.

[19] R. Banerjee and S. Ghosh, Seiberg-Witten map and the axial anomaly in noncommutative field theory, Phys. Lett. B 533 (2002) 162 hep-th/0110177.

[20] R. Banerjee, Anomalies in noncommutative gauge theories, Seiberg-Witten transformation and Ramond-Ramond couplings, hep-th/0301174.

[21] B.L. Cerchiai, A.F. Pasqua and B. Zumino, The Seiberg-Witten map for noncommutative gauge theories, hep-th/0206231.

[22] P. Breitenlohner and D. Maison, Dimensional renormalization and the action principle, Commun. Math. Phys. 52 (1977) 11.

[23] G. Barnich, F. Brandt and M. Grigoriev, Seiberg-Witten maps and noncommutative Yang-Mills theories for arbitrary gauge groups, J. High Energy Phys. 08 (2002) 023 hep-th/0206003.

[24] H. Georgi and S.L. Glashow, Gauge theories without anomalies, Phys. Rev. D 6 (1972) 429.

[25] J.M. Gracia-Bondia and C.P. Martin, Chiral gauge anomalies on noncommutative $R^{4}$, Phys. Lett. B 479 (2000) 321 hep-th/0002171.

[26] L. Bonora, M. Schnabl and A. Tomasiello, A note on consistent anomalies in noncommutative YM theories, Phys. Lett. B 485 (2000) 311 hep-th/0002210.

[27] D.J. Gross and R. Jackiw, Effect of anomalies on quasirenormalizable theories, Phys. Rev. D 6 (1972) 477.

[28] F. Brandt, N. Dragon and M. Kreuzer, Completeness and nontriviality of the solutions of the consistency conditions, Nucl. Phys. B 332 (1990) 224. 